\title{
Power Muirhead Mean Operators of Interval-Valued Intuitionistic Fuzzy Values in the Framework of Dempster-Shafer Theory for Multiple Criteria Decision-Making
}

\section{Yanru Zhong}

GUET: Guilin University of Electronic Technology

Huanan Zhang (D2571520308@qq.com )

GUET: Guilin University of Electronic Technology

\section{Liangbin Cao}

GUET: Guilin University of Electronic Technology

\section{Yiyuan Li}

GUET: Guilin University of Electronic Technology https://orcid.org/0000-0003-1362-2610

\section{Yuchu Qin}

University of Huddersfield School of Computing and Engineering

Xiaonan Luo

GUET: Guilin University of Electronic Technology

\section{Research Article}

Keywords: Interval-valued intuitionistic fuzzy value, Dempster-Shafer theory, Multiple criteria decisionmaking, Power average operator, Muirhead mean operator

Posted Date: February 1st, 2022

DOI: https://doi.org/10.21203/rs.3.rs-1038177/v1

License: (c) (i) This work is licensed under a Creative Commons Attribution 4.0 International License. Read Full License 


\title{
Power Muirhead Mean Operators of Interval-Valued Intuitionistic Fuzzy Values in the Framework of Dempster-Shafer Theory for Multiple Criteria Decision-Making
}

Yanru Zhong a, Huanan Zhang ${ }^{\mathrm{a}}$, Liangbin Cao ${ }^{\mathrm{a}}$, Yuchu Qin ${ }^{\mathrm{b}}$, Yiyuan $\mathrm{Li}^{\mathrm{a},{ }^{*}}$, Xiaonan Luo ${ }^{\mathrm{a}}$

${ }^{*}$ Corresponding author.

E-mail address: lyy@guet.edu.cn (Y. Li).

${ }^{\text {a }}$ Guangxi Key Laboratory of Intelligent Processing of Computer Images and Graphic, Guilin University of Electronic Technology, Guilin 541004, China

${ }^{\mathrm{b}}$ School of Computing and Engineering, University of Huddersfield, Huddersfield HD1 3DH, UK

\begin{abstract}
:
There have been many aggregation operators of interval intuitionistic fuzzy values (IVIFVs) for solving multiple criteria decision-making problems with IVIFVs. Each of these operators can work well in its specific context. However, they could generate irrational sorting results because of a few limitations. In this paper, two novel power Muirhead mean operators of IVIFVs under the Dempster-Shafer theory framework which are free of the limitations of existing operators of IVIFVs are presented. The expressions of these operators are first constructed. Then their mathematical properties are studied and proved. After that, a method to solve multiple criteria decision-making problems with IVIFVs is proposed based on the presented operators. Finally, a practical example is introduced to illustrate the application of the proposed method and several qualitative comparisons to some existing operators of IVIFVs are carried out to demonstrate the effectiveness and advantages of the proposed method.
\end{abstract}

Keywords: Interval-valued intuitionistic fuzzy value · Dempster-Shafer theory · Multiple criteria decisionmaking $\cdot$ Power average operator $\cdot$ Muirhead mean operator

\section{Introduction}

Multiple-criteria decision-making (MCDM) is the process to rank desirable alternatives through explicitly evaluating multiple criteria (Pedrycz \& Chen, 2015). To determine desirable alternatives, MCDM generally includes two critical tasks (Greco, Figueira, \& Ehrgott, 2016), specifically (1) quantifying the considered criteria of each alternative and (2) synthetically aggregating the performance of each alternative with respect to the quantified criteria.

Since the increasing complexity of decision-making problems introduce more uncertain information in real world, it is quite difficult to quantify criteria of each alternative with exact numbers. To effectively deal with uncertain information, Zedeh (1996) proposed an important tool, named fuzzy set, which introduces membership degrees to quantify the considered criteria and is sufficient for certain applications (Fu, Miao \& Wu, 2021; Pramanik, Baidya \& Dhang, 2021). However, only using membership degrees as criteria cannot separately express the negative and refusal degrees. To this end, Atanassov (1986) proposed intuitionistic fuzzy set (IFS), which also considers a non-membership degree to further extend fuzzy set. Furthermore, Atanassov (1999) introduced interval-valued intuitionistic fuzzy set (IVIFS), where membership degree and non-membership degree are both represented by intervals. Such interval can measure objectively the neutral hesitancy degree of the evaluators. Because of such capability, various uncertain decision making fields based on IVIFS are emerged, e.g., multi-objective planning (Liu et al., 2017; Chen \& Kuo, 2018; Garg \& Arora, 2018), sentiment classification (Einstein, Albert, Boris \& Nathan, 1935), cluster analysis (Xu, 2009), and multi-attribute decision-making( Zindani, Maity \& Bhowmik, 2020; Oztaysi et al., 2017; Shen, Huang, Liu, Jiang \& Zhao, 2020). There are many works focusing on the theory and application of IVIFS, which mainly include the following three aspects: (1) Basic theory, such as operation criterion (Xu, 2007; Xu, 2007), score function (Xu, 2007; Hung \& Wu, 2002; Garg \& Kumar, 2020), distance measurement (Xu, 2010; Garg \& Kumar, 2020), and similarity measurement (Ren \& Wang, 2015). (2) Extended MCDM methods for IVIFS, such as multi-objective linear/nonlinear programming (Mesa, Fabio, José, Merigó \& Anna, 2017) and statistical convergent sequence spaces. (3) Aggregation 
operators to aggregating IVIFVs, such as power average operator (He et al., 2013), Muirhead mean operator (Muirhead, 1902), Maclaurin symmetric mean operator (Sun, Gang \& Xia, 2016), power Bonferroni mean operator (Liu \& Li, 2017), and Heronian mean operator (Yu \& Wu, 2012).

Another critical task of MCDM methods is to efficently generate a ranking performance of each alternative. Since aggregation operator can integrate the quantified criteria from various indicators to get synthetical scores and the final ranking can be obtained through these scores. Therefore, aggregation operators are of great importance for solving MCDM problems, which performs better than conventional decision-making methods (e.g., TOPSIS (Li, Mao, Lei, Gao \& Liu, 2020; Zhang, Zhan \& Yao, 2020; Chen, 2015; Garg \& Kaur, 2019), VIKOR (Zeng, Chen \& Kuo, 2019; Li \& Jiang, 2011), ELECTRE (Veeramachaneni \& Kandikonda, 2016), BDCI (Meng, Chen \& Tang, 2021), and new score function (Chen \& Tsai, 2021; Kumar \& Chen, 2021)). When using aggregation operators, it is of necessity to reduce the effect of unreasonable input data on the aggregations results. Yager (Yager \& Ronald, 2001) originally proposed the power average (PA) operator. In the PA operator, the distance between parameters is used to calculate the positive correlations of parameters, and these positive correlations are accumulated to adjust final weights. Through such operation, the impact of unreasonable data on the sorting results can be weakened (Liu, Chen \& Wang, 2018). In addition to the influence of unreasonable data, the relationship between parameters is also an important factor to be considered. The Muirhead mean (MM) operator is well-known for having the capability to capture the interrelationships among all aggregation parameters (Muirhead, 1902; Garg, Mahmood, Ahmmad, Khan \& Ali, 2021). For these reasons, it would be of significance to combine the PA operator with the MM operator, which is generally called power Muirhead mean (PMM) operator (Khan, Hassan \& Mahmood, 2018). Xu and Shang (2019) extended the combined PMM operator to aggregate interval-valued intuitionistic fuzzy values and developed interval-valued intuitionistic fuzzy power Muirhead mean (IVIFPMM) operator.

Dempster-Shafer theory (DST) was first proposed by Dempster (Shafer \& Glenn, 1976; Dempster, 1967), and was further extended by Shafer (Dempster \& Arthur, 2008). DST has a great advantage in dealing with inaccurate or vague information (Liu \& Gao, 2019; Gao, Zhang \& Liu, 2019; Qin et al., 2020; Zhong et al., 2021). Under the DST framework, the basic probability assignment (BPA) is used to represent the probability of occurrence of the standard in a basic event, while the confidence function and likelihood function are used to form belief interval. The confidence function and likelihood function represent the focal element confidence and uncertainty in the event, respectively. As mentioned in (Dymova, Ludmila \& Pavel, 2016), interval intuitionistic fuzzy values (IVIFVs) in the DST framework still contain original fuzzy information (i.e., membership degree, non-membership degree and a hesitancy degree). IVIFVs under the DST framework are found to be get rid of the shortcomings and limitations of IVIFPMM. Thus, it is a good way to aggregate IVIFVs under the DST framework, and thus obtain more reasonable aggregation results.

Based on the background described above, the motivations of this paper are summarized as follows:

(1) To measure the neutral hesitancy degree, we use interval-valued intuitionistic fuzzy set (IVIFS) in the field of MCDM. To concurrently maintain the specific advantage of PA operator and MM operator, two operators are combined to aggregate IVIFVs. The combined power Muirhead mean (PMM) operators are robust to outliers and reduce the associated values on sorting results (Liu \& Liu, 2019; Khan, Hassan \& Mahmood, 2018).

(2) To improve the above PMM operators of IVIFVs, the Dempster-Shafer theory (DST) are introduced to develop novel PMM operators of IVIFVs. The operational laws of intuitionistic fuzzy values under the DST framework are found to be free of the limitations of the operational laws based on Archimedean t-norm and t-conorm (Qin et al., 2020). The IVIFPMM under the DST framework based on such basic laws tend to generate more reasonable and convincing results for an MCDM problem (Qin et al., 2020; Liu \& Gao, 2019)

(3) To consider the relative importance of the input criteria, weights are introduced and combined into the operator. The weighted operators have the capability of reducing the influence of unreasonable aggregation values on the final 
aggregated result. Further, the weights can be also expressed by IVIFVs, so the MCDM problems can be solved without deblurring fuzzy information of weights.

Based on the motivations above, this paper aims to develop a set of novel operational laws of IVIFVs under the DST framework and construct PMM operators of IVIFVs based on these operational laws for dealing with the MCDM problems. This aim is achieved via combining the MM operator and the PA operator to assess the weighted criteria of IVIFVs under the DST framework. The major contributions of the paper are summarized as follows: (1) proposes $I_{\text {IVIFPM }} M_{D S T}$ and IVIFWPMM $M_{D S T}$ (the weight $W$ can be taken as a constant or a IVIFV) operators which can maintain the common advantages of the existing PMM operator and concurrently generate more reasonable and convincing results; (2) develops and proves basic properties (Idempotence, Commutativity, and Boundedness) of the proposed operators; (3) Proposes a method based on the proposed operators to solve the MCDM problems with IVIFVs. The proposed method is compared with other existing MCDM methods to show its advantages.

The rest of the paper is organized as follows: In Section 2, some basic concepts and related operations of IVIFVs are introduced, the limitations of basic algorithm of IVIFVs are highlighted, and the definition and operations of IVIFVs under the DST framework are recalled. Section 3 presents the IFIVPMM and IVIFWPMM operators under the DST framework. In Section 4, a method based on the presented operators to solve the MCDM problems with IVIFVs is proposed. In Section 5, a set of comparative experiments for illustrating the rationality and advantages of the proposed method are documented. Section 6 ends the paper with a conclusion.

\section{Preliminaries}

\subsection{IVIFSs}

Definition 2.1 (Atanassov \& Krassimir, 1999) Let E be the set of natural numbers, then an IVIFS named G, defined on $\mathrm{E}$, can be expressed as:

$$
G=\left\{<e, u_{G}(e), v_{G}(e)>\mid e \in E\right\},
$$

where, $u_{G}(e) \subset[0,1]$ and $v_{G}(e) \subset[0,1]$ (where $e \in E$ and $u_{G}(e)+\sup \left(v_{G}(e)\right) \leq 1$ ). We will use $G=\left\{\left[u_{G}{ }^{L}, u_{G}{ }^{U}\right],\left[v_{G}{ }^{L}, v_{G}{ }^{U}\right]\right\}$ to denote an IVIFV for convenience. Where, $\left[u_{G}{ }^{L}, u_{G}{ }^{U}\right]$ denotes the degree of membership interval of $G$, and $\left[v_{G}{ }^{L}, v_{G}{ }^{U}\right]$ is the non-membership interval of $\mathrm{G}$.

$\mathrm{Xu}(\mathrm{Xu}, 2007)$ proposed a set of arithmetic operations of IVIFVs, which are defined below:

Definition 2.2 (Xu, 2007) Let $\mathrm{G}=\left\{\left[\mathrm{u}_{\mathrm{G}}{ }^{\mathrm{L}}, \mathrm{u}_{\mathrm{G}}{ }^{\mathrm{U}}\right],\left[\mathrm{v}_{\mathrm{G}}^{\mathrm{L}}, \mathrm{v}_{\mathrm{G}}^{\mathrm{U}}\right]\right\}$ and $\mathrm{I}=\left\{\left[\mathrm{u}_{\mathrm{I}}^{\mathrm{L}}, \mathrm{u}_{\mathrm{I}}^{\mathrm{U}}\right],\left[\mathrm{v}_{\mathrm{I}}^{\mathrm{L}}, \mathrm{v}_{\mathrm{I}}^{\mathrm{U}}\right]\right\}$ be any two IVIFVs, then:

$$
\begin{aligned}
G \oplus I & =\left\{\left[u_{G}^{L}+u_{I}^{L}-u_{G}^{L} u_{I}^{L}, u_{G}^{U}+u_{I}^{U}-u_{G}^{U} u_{I}^{U}\right],\left[v_{G}^{L} v_{I}^{L}, v_{G}^{U} v_{I}^{U}\right]\right\}, \\
G \otimes I & =\left\{\left[u_{G}^{L} u_{I}^{L}, u_{G}^{U} u_{I}^{U}\right],\left[v_{G}^{L}+v_{I}^{L}-v_{G}^{L} v_{I}^{L}, v_{G}^{U}+v_{I}^{U}-v_{G}^{U} v_{I}^{U}\right]\right\}, \\
\lambda G & =\left\{\left[1-\left(1-u_{G}^{L}\right)^{\lambda}, 1-\left(1-u_{G}^{U}\right)^{\lambda}\right],\left[\left(v_{G}^{L}\right)^{\lambda},\left(v_{G}^{U}\right)^{\lambda}\right]\right\}, \\
G^{\lambda} & =\left\{\left[\left(u_{G}^{L}\right)^{\lambda},\left(u_{G}^{U}\right)^{\lambda}\right],\left[1-\left(1-v_{G}^{L}\right)^{\lambda}, 1-\left(1-v_{G}^{U}\right)^{\lambda}\right]\right\},
\end{aligned}
$$

where $\lambda>0$.

Based on the arithmetic operations above, an interval-valued intuitionistic fuzzy weighted arithmetic mean (IVIFWAM) operator and an interval-valued intuitionistic fuzzy weighted geometric mean (IVIFWGM) operator were presented in (Xu, 2007; Xu, 2007).

Definition $2.3(\mathrm{Xu}, 2007 ; \mathrm{Xu}, 2007)$ Let $G_{i}(i=1,2,3 \ldots, n)$ be $n$ IVIFVs and $w=\left(w_{1}, w_{2}, \ldots, w_{n-1}, w_{n}\right)^{T}$ be the weight corresponding to $G_{i}(i=1,2,3 \ldots, n)$, where $w_{i} \in[0,1]$ and $\sum_{i=1}^{n} w_{i}=1$. The IVIFWAM operator maps $G^{n}$ to $G$, defined as follows: 


$$
\begin{array}{r}
\operatorname{IVIFWAM}\left(G_{1}, G_{2}, \ldots, G_{n}\right) \\
=\left\{\left[1-\prod_{i=1}^{n}\left(1-u_{G_{i}}^{L}\right)^{w_{i}}, 1-\prod_{i=1}^{n}\left(1-u_{G_{i}}^{U}\right)^{w_{i}}\right],\left[\prod_{i=1}^{n}\left(v_{G_{i}}^{L}\right)^{w_{i}}, \prod_{i=1}^{n}\left(v_{G_{i}}^{U}\right)^{w_{i}}\right]\right\}
\end{array}
$$

Definition 2.4 (Xu, 2007; Xu, 2007) Let $G_{i}(i=1,2,3 \ldots, n)$ be $n$ IVIFVs and $w=\left(w_{1}, w_{2}, \ldots, w_{n-1}, w_{n}\right)^{T}$ be the weight corresponding to $G_{i}(i=1,2,3 \ldots, n)$, where $w_{i} \in[0,1]$ and $\sum_{i=1}^{n} w_{i}=1$. The IVIFWGM operator maps $G^{n}$ to $G$, i.e.,

$\operatorname{IVIFWGM}\left(G_{1}, G_{2}, \ldots, G_{n}\right)$

$$
=\left\{\left[\prod_{i=1}^{n}\left(u_{G_{i}}^{L}\right)^{w_{i}}, \prod_{i=1}^{n}\left(u_{G_{i}}^{U}\right)^{w_{i}}\right],\left[1-\prod_{i=1}^{n}\left(1-v_{G_{i}}^{L}\right)^{w_{i}}, 1-\prod_{i=1}^{n}\left(1-v_{G_{i}}^{U}\right)^{w_{i}}\right]\right\}
$$

To compare two IVIFVs, an accuracy function and a score function were introduced in (Xu, 2007).

Definition 2.5 (Xu, 2007) The score function and accuracy function of an IVIFV G are respectively defined as follows:

$$
\begin{gathered}
S F(G)=\frac{u_{G}^{L}+u_{G}^{U}-v_{G}^{L}-v_{G}^{U}}{2}, S F(G) \in[-1,1], \\
A F(G)=\frac{u_{G}^{L}+u_{G}^{U}+v_{G}^{L}+v_{G}^{U}}{2}, A F(G) \in[0,1] .
\end{gathered}
$$

The order relation of any two IVIFVs (e.g. $\mathrm{G}$ and $\mathrm{H}$ ) can be obtained from the following rules:

(1) if $\operatorname{SF}(\mathrm{G})>\mathrm{SF}(\mathrm{H})$, then $\mathrm{G}>\mathrm{H}$.

(2) if $\mathrm{SF}(\mathrm{G})=\mathrm{SF}(\mathrm{H})$, then :

(i) if $\mathrm{AF}(\mathrm{G})>\mathrm{AF}(\mathrm{H})$, then $\mathrm{G}>\mathrm{H}$.

(ii) if $\mathrm{AF}(\mathrm{G})=\mathrm{AF}(\mathrm{H})$, then $\mathrm{G}=\mathrm{H}$.

(iii) if $\mathrm{AF}(\mathrm{G})<\mathrm{AF}(\mathrm{H})$, then $\mathrm{G}<\mathrm{H}$.

(3) if $\mathrm{SF}(\mathrm{G})<\mathrm{SF}(\mathrm{H})$, then $\mathrm{G}<\mathrm{H}$.

Let $\mathrm{G}_{1}, \mathrm{G}_{2}$ be two IVIFVs, it is easy to prove that Eqs. (2) - (5) have the following properties:

(1) $\mathrm{G}_{1} \oplus \mathrm{G}_{2}=\mathrm{G}_{2} \oplus \mathrm{G}_{1}$,

(2) $\mathrm{G}_{1} \otimes \mathrm{G}_{2}=\mathrm{G}_{2} \otimes \mathrm{G}_{1}$,

(3) $\lambda\left(G_{1} \oplus G_{2}\right)=\lambda G_{1} \oplus \lambda G_{2}$, where $\lambda>0$

(4) $(\lambda+\mu) \mathrm{G}_{1}=\lambda \mathrm{G}_{1} \oplus \mu \mathrm{G}_{1}$, where $\lambda, \mu>0$

(5) $\left(\mathrm{G}_{1} \otimes \mathrm{G}_{2}\right)^{\lambda}=\mathrm{G}_{1}{ }^{\lambda} \otimes \mathrm{G}_{2}{ }^{\lambda}$, where $\lambda>0$

(6) $\mathrm{G}_{1}{ }^{\lambda} \otimes \mathrm{G}_{1}{ }^{\mu}=\mathrm{G}_{1}{ }^{\lambda+\mu}$, where $\lambda, \mu>0$

The studies in (Xu, 2007; Xu, 2007) showed that some limitations of Eqs. (2) - (5) could lead to unreasonable results when applying them to solve the MCDM problems. Some intuitive examples are as follows:

Example 2.1 Let $\mathrm{g} 1=\{[0.4,0.6],[0.1,0.3]\}, \mathrm{g} 2=\{[0.3,0.5],[0,0.1]\}$ and $\mathrm{g} 3=\{[0.1,0.3],[0.1,0.3]\}$. From Definition 2.2, we can obtain that $\mathrm{g} 1 \oplus \mathrm{g} 3=\{[0.46,0.72],[0.01,0.09]\}, \mathrm{g} 2 \oplus \mathrm{g} 3=\{[0.37,0.65],[0,0.03]\}$. Then it is easy to obtain that $\mathrm{SF}(\mathrm{g} 1)=0.3, \mathrm{SF}(\mathrm{g} 2)=0.35$, and $\mathrm{g} 1<\mathrm{g} 2$, while $\mathrm{SF}(\mathrm{g} 1 \oplus \mathrm{g} 3)=0.54, \mathrm{SF}(\mathrm{g} 2 \oplus \mathrm{g} 3)=0.495$, and $\mathrm{g} 1 \oplus \mathrm{g} 3>\mathrm{g} 2 \oplus \mathrm{g} 3$. Hence, we can conclude that we cannot always get 'g1 $\oplus \mathrm{g} 3>\mathrm{g} 2 \oplus \mathrm{g} 3$ 'from 'g1 > g2'.

Example 2.2 Let $\mathrm{g} 1=\{[0,0.2],[0.3,0.4]\}, \mathrm{g} 2=\{[0.2,0.4],[0.4,0.6]\}$ and $\mathrm{g} 3=\{[0.2,0.4],[0.2,0.4]\}$, we can get that $\mathrm{g} 1 \otimes \mathrm{g} 3=\{[0,0.08],[0.44,0.64]\}, \mathrm{g} 2 \otimes \mathrm{g} 3=\{[0.04,0.16],[0.52,0.76]\}$. Then it is easy to obtain that $\mathrm{SF}(\mathrm{g} 1)$ 
$=-0.25, \quad \mathrm{SF}(\mathrm{g} 2) \quad=-0.2, \quad$ and $\quad \mathrm{g} 1 \quad<\mathrm{g} 2, \quad$ while $\quad \mathrm{SF}(\mathrm{g} 1 \otimes \mathrm{g} 3)=-0.5 \quad, \quad \mathrm{SF}(\mathrm{g} 2 \otimes \mathrm{g} 3)=-0.54 \quad, \quad$ and $\mathrm{g} 1 \otimes \mathrm{g} 3>\mathrm{g} 2 \otimes \mathrm{g} 3$. Therefore, we can conclude that we cannot always get 'g1 $\otimes \mathrm{g} 3>\mathrm{g} 2 \otimes \mathrm{g} 3$ 'from 'g1 > g2'.

Example 2.3 Let $\mathrm{g} 1=\{[0.3,0.5],[0.2,0.4]\}, \mathrm{g} 2=\{[0.2,0.4],[0.1,0.4]\}$ and $\lambda=0.5$. From Definition 2.2, we can get that $\lambda \mathrm{g} 1=\{[0.1633,0.2929],[0.4472,06325]\}, \lambda \mathrm{g} 2=\{[0.1056,0.2254],[0.3162,0.6325]\}$. Then it is easy to obtain that $\mathrm{SF}(\mathrm{g} 1)=0.1, \mathrm{SF}(\mathrm{g} 2)=0$, and $\mathrm{g} 1>\mathrm{g} 2$, but $\mathrm{SF}(\lambda \mathrm{g} 1)=-0.6235, \mathrm{SF}(\lambda \mathrm{g} 2)=-0.6177$, and $\lambda \mathrm{g} 1<\lambda \mathrm{g} 2$. Therefore, we can conclude that we cannot always get ' $\lambda \mathrm{g} 1>\lambda \mathrm{g} 2$ ' from ' $\mathrm{g} 1>\mathrm{g} 2$ '.

Example 2.4 Let $\mathrm{g} 1=\{[0.3,0.5],[0.2,0.4]\}, \mathrm{g} 2=\{[0.2,0.4],[0.1,0.4]\}$ and $\lambda=0.5$. We can obtain g1 $1^{\lambda}=$ $\{[0.4472,06325],[0.1633,0.2929]\}, \mathrm{g} 2^{\lambda}=\{[0.3162,0.6325],[0.1056,0.2254]\}$. Then, $\mathrm{SF}(\mathrm{g} 1)=-0.1, \mathrm{SF}(\mathrm{g} 2)=0$, and $\mathrm{g} 1<\mathrm{g} 2$, while $\mathrm{SF}\left(\mathrm{g} 1^{\lambda}\right)=0.6235, \mathrm{SF}\left(\mathrm{g} 2^{\lambda}\right)=0.6177$, and $\mathrm{g} 1^{\lambda}>\mathrm{g} 2^{\lambda}$. Therefore, we can conclude that we cannot always get ' $\mathrm{g} 1^{\lambda}>\mathrm{g} 2^{\lambda 1}$ when ' $\mathrm{g} 1>\mathrm{g} 2^{\prime}$.

The above four examples show some limitations of the arithmetic operations in Eqs. (2) - (5), which could lead to irrational results when dealing with MCDM problems. Further, (Dymova, Ludmila \& Pavel, 2016) shows that there is a very close relationship between DST and IVIFV. Under the DST framework, an IVIFV has transparent and fruitful semantics. More importantly, the arithmetic operations of IVIFVs under this framework can overcome the limitations above.

\subsection{IFIVS in the framework of DST}

The recognition framework, marked as $\Delta$, is a finite mutually exclusive set of $q$ propositional hypotheses, i.e., $\Delta=$ $\{1,2, \ldots, \mathrm{q}\}$. Let $\mathrm{P}(\Delta)$ be the power set of $\Delta$, then $\mathrm{P}(\Delta)=\{\phi, 1,2,3, \ldots, q,(q-1, q),(\mathrm{q}-1, \mathrm{q}-2), \ldots,(1,2),(1,2,3), \ldots, \Delta\}$. It is obvious that $\mathrm{P}(\Delta)$ contains $2^{q}$ elements.

A basic probability assignment (BPA), mapping $\mathrm{P}(\Delta)$ to [0,1], satisfies $\sum_{X \subseteq \mathrm{P}(\Delta)} \mathrm{m}(X)=1$ and $m(\phi)=0$. Herein, $\mathrm{m}(X)$ represents the degree to which the hypothesis or proposition belongs to X. The element in $\Delta$ and BPA that is not 0 is called the focus element.

Definition 2.6 (Dempster \& Arthur, 2008; Mesa, Fabio, José, Merigó \& Anna, 2017) Let $m$ be a BPA with a domain of $\Delta$. The belief function (Bel) can be expressed as:

$$
\operatorname{Bel}(\mathrm{X})=\sum_{S \subseteq X} m(S)
$$

where $m(S)>0$

Definition 2.7 (Dempster \& Arthur, 2008; Mesa, Fabio, José, Merigó \& Anna, 2017) Let $m$ be a BPA with a domain of $\Delta$. The plausibility function $(\mathrm{Pl})$ can be expressed as:

$$
P l(X)=\sum_{S \cap X \neq \phi} m(S)=1-\operatorname{Bel}(\bar{S}),
$$

where $\bar{S}$ denotes the complementary collection of X.

From the above definition of $\mathrm{Bel}$ and $\mathrm{Pl}$, a belief interval $(\mathrm{BI})$ can be formed, that is, $\mathrm{BI}(\mathrm{X})=[\mathrm{Bel}(\mathrm{X}), \mathrm{Pl}(\mathrm{X})]$. Specifically, BI is composed of the "true, probability" interval of X.

Based on the definitions above, the definition of IVIFS under the DST framework is as follow :

Definition 2.8 (Dymova, Ludmila \& Pavel, 2016) Let $G$ be an IVIFS based on a generic finite set $S$, then:

$$
G=\left\{\left\langle s,\left[B I_{G}(s)\right]\right\rangle \mid s \in S\right\},
$$

where

$$
\left[B I_{G}(s)\right]=\left[B I_{G}^{L}(s), B I_{G}^{U}(s)\right],
$$

is a belief interval with a bound of BIs :

$$
\begin{aligned}
B I_{G}^{L}(s) & =[\inf \mu(s), 1-\sup v(s)], \\
B I_{G}^{U}(s) & =[\sup \mu(s), 1-\inf v(s)],
\end{aligned}
$$


where $\mu(s), v(s) \subset[0,1]$ and inf $\mu(s)+\sup v(s) \leq 1, \sup \mu(s)+\inf v(s) \leq 1$.

Definition 2.9 (Dymova, Ludmila \& Pavel, 2016) The expression form of an IVIFV G under the DST framework is:

$$
G=\left[B I_{G}\right]=\left[B I_{G}^{L}, B I_{G}^{U}\right],
$$

where

$$
\begin{gathered}
B I_{G}^{L}(x)=\left[\operatorname{Bel}_{G}^{L}, P l_{G}^{L}\right]=\left[\mu_{G}^{L}, 1-v_{G}^{U}\right], \\
B I_{G}^{U}(x)=\left[\operatorname{Bel}_{G}^{U}, P l_{G}^{U}\right]=\left[\mu_{G}^{U}, 1-v_{G}^{L}\right],
\end{gathered}
$$

where $\mu_{G}^{L}, \mu_{G}^{U}, v_{G}^{L}, v_{G}^{U} \in[0,1]$ and $\mu_{G}^{L}+v_{G}^{U} \leq 1, \mu_{G}^{U}+v_{G}^{L} \leq 1$.

On the basis of the definition above, the arithmetic operations of IVIFVs under the DST framework are defined as follows:

Definition 2.10 (Dymova, Ludmila \& Pavel, 2016) Let $G=\left\{\left[\operatorname{Bel}_{G}^{L}, \mathrm{Pl}_{G}^{L}\right],\left[\operatorname{Bel}_{G}^{U}, \mathrm{Pl}_{G}^{U}\right]\right\}$ and $\mathrm{I}=\left\{\left[\operatorname{Bel}_{I}^{L}, \mathrm{Pl}_{I}^{\mathrm{L}}\right],\left[\mathrm{Bel}_{\mathrm{I}}^{\mathrm{U}}, \mathrm{Pl}_{\mathrm{I}}^{U}\right]\right\}$ be two BIs, Let $\mathrm{H}=[\mathrm{Bel}, \mathrm{Pl}]$ be a BI, then:

$$
\begin{aligned}
& G \oplus_{B N P} I=\left\{\left[\frac{\mathrm{Bel}_{\mathrm{G}}^{\mathrm{L}}+\mathrm{Bel}_{\mathrm{L}}^{\mathrm{L}}}{2}, \frac{\mathrm{Pl}_{\mathrm{G}}^{\mathrm{L}}+\mathrm{Pl}_{\mathrm{L}}^{\mathrm{L}}}{2}\right],\left[\frac{\mathrm{Bel}_{\mathrm{G}}^{\mathrm{U}}+\mathrm{Bel}_{\mathrm{I}}^{\mathrm{U}}}{2}, \frac{\mathrm{Pl}_{\mathrm{G}}^{\mathrm{U}}+\mathrm{Pl}_{\mathrm{I}}^{\mathrm{U}}}{2}\right]\right\}, \\
& G \bigotimes_{B N P} I=\left\{\left[\operatorname{Bel}_{\mathrm{G}}^{\mathrm{L}} \operatorname{Bel}_{\mathrm{I}}^{\mathrm{L}}, \mathrm{Pl}_{\mathrm{G}}^{\mathrm{L}} \mathrm{Pl}_{\mathrm{I}}^{\mathrm{L}}\right],\left[\operatorname{Bel}_{\mathrm{G}}^{\mathrm{U}} \operatorname{Bel}_{\mathrm{I}}^{\mathrm{U}}, \mathrm{Pl}_{\mathrm{G}}^{\mathrm{U}} \mathrm{Pl}_{\mathrm{I}}^{\mathrm{U}}\right]\right\}, \\
& \lambda G=\left\{\left[\lambda \mathrm{Bel}_{\mathrm{G}}^{\mathrm{L}}, \lambda \mathrm{Pl}_{\mathrm{G}}^{\mathrm{L}}\right],\left[\lambda \mathrm{Bel}_{\mathrm{G}}^{\mathrm{U}}, \lambda \mathrm{Pl}_{\mathrm{G}}^{\mathrm{U}}\right]\right\}, \\
& G^{\lambda}=\left\{\left[\left(\operatorname{Bel}_{G}^{\mathrm{L}}\right)^{\lambda},\left(\mathrm{Pl}_{\mathrm{G}}^{\mathrm{L}}\right)^{\lambda}\right],\left[\left(\mathrm{Bel}_{\mathrm{G}}^{\mathrm{U}}\right)^{\lambda},\left(\mathrm{Pl}_{\mathrm{G}}^{\mathrm{U}}\right)^{\lambda}\right]\right\} \text {, } \\
& G^{H}=\left\{\left[\left(\operatorname{Bel}_{\mathrm{G}}^{\mathrm{L}}\right)^{P l},\left(\mathrm{Pl}_{\mathrm{G}}^{\mathrm{L}}\right)^{B e l}\right],\left[\left(\operatorname{Bel}_{\mathrm{G}}^{\mathrm{U}}\right)^{P l},\left(\mathrm{Pl}_{\mathrm{G}}^{\mathrm{U}}\right)^{B e l}\right]\right\}, \\
& G^{I}=\left\{\left[\left(\operatorname{Bel}_{\mathrm{G}}^{\mathrm{L}}\right)^{P l_{I}^{U}},\left(\mathrm{Pl}_{\mathrm{G}}^{\mathrm{L}}\right)^{B e l_{I}^{L}}\right],\left[\left(\mathrm{Bel}_{\mathrm{G}}^{\mathrm{U}}\right)^{P l_{I}^{U}},\left(\mathrm{Pl}_{\mathrm{G}}^{\mathrm{U}}\right)^{B e l_{I}^{L}}\right]\right\}, \\
& H \otimes_{B N P} G=\left\{\left[\mathrm{Bel} \mathrm{Bel}_{\mathrm{I}}^{\mathrm{L}}, \mathrm{Bel} \mathrm{Pl} \mathrm{L}\right],\left[\mathrm{Pl} \mathrm{Bel}_{\mathrm{I}}^{\mathrm{U}}, \mathrm{Pl} \mathrm{Pl} \mathrm{I}_{\mathrm{I}}^{\mathrm{U}}\right]\right\},
\end{aligned}
$$

From the arithmetic operations above, an IVIFWAM $M_{D S T}$ operator and an IVIFWGM of DST can be deduced below (Dymova, Ludmila \& Pavel, 2016):

Let $G_{i}=\left\{\left[\mathrm{Bel}_{\mathrm{G}_{\mathrm{i}}}^{\mathrm{L}} \mathrm{Pl}_{\mathrm{G}_{\mathrm{i}}}^{\mathrm{L}}\right],\left[\mathrm{Bel}_{\mathrm{G}_{\mathrm{i}}}^{\mathrm{U}}, \mathrm{Pl}_{\mathrm{G}_{\mathrm{j}}}^{\mathrm{U}}\right]\right\}(I=1,2,3 \ldots, n)$ be a set of BIs $. w=\left(w_{1}, w_{2}, \ldots, w_{n-1}, w_{n}\right)^{T}$ is the weight corresponding to $G_{i}(i=1,2,3 \ldots, n)$, which satisfies: $w_{i} \in[0,1]$ and $\sum_{i=1}^{n} w_{i}=1$. Then the IVIFWAM operator in the framework of DST is given as follows:

$$
\operatorname{IVIWAM}_{D S T}\left(G_{1}, G_{2}, \ldots, G_{n}\right)=\left\{\left[\sum_{i=1}^{n} w_{i} \operatorname{Bel}_{\mathrm{G}_{\mathrm{i}}}^{\mathrm{L}}, \sum_{i=1}^{n} w_{i} \mathrm{Pl}_{\mathrm{G}_{\mathrm{i}}}^{\mathrm{L}}\right],\left[\sum_{i=1}^{n} w_{i} \mathrm{Bel}_{\mathrm{G}_{\mathrm{i}}}^{\mathrm{U}}, \sum_{i=1}^{n} w_{i} \mathrm{Pl}_{\mathrm{G}_{\mathrm{i}}}^{\mathrm{U}}\right]\right\}
$$

The IVIFWGM operator in the framework of DST is given as follow:

$$
\operatorname{IVIWGM}_{D S T}\left(G_{1}, G_{2}, \ldots, G_{n}\right)=\left\{\left[\prod_{k=1}^{n}\left(\operatorname{Bel}_{G_{k}}^{L}\right)^{w_{k}}, \prod_{k=1}^{n}\left(P l_{G_{k}}^{L}\right)^{w_{k}}\right],\left[\prod_{k=1}^{n}\left(\operatorname{Bel}_{G_{k}}^{U}\right)^{w_{k}}, \prod_{k=1}^{n}\left(P l_{G_{k}}^{U}\right)^{w_{k}}\right]\right\}
$$

An accuracy function and a score function are important tools for comparing IVIFVs in the framework of DST, which are defined as follows (Dymova, Ludmila \& Pavel, 2016):

$$
\begin{gathered}
S F(G)=\frac{B e l_{G}^{L}+B e l_{G}^{U}+P l_{G}^{L}+P l_{G}^{U}-2}{2}, S F(G) \in[-1,1], \\
A F(G)=\frac{B e l_{G}^{L}+B e l_{G}^{U}-P l_{G}^{L}-P l_{G}^{U}+2}{2}, H(G) \in[0,1] .
\end{gathered}
$$

Let $G_{1}, G_{2}$ be two BIs. It is easy to prove that Eqs.(25) - (31) has the following properties:

$$
\begin{aligned}
& \text { (1) } G_{1} \oplus G_{2}=G_{2} \oplus G_{1}, \\
& \text { (2) } G_{1} \otimes G_{2}=G_{2} \otimes G_{1} \text {, }
\end{aligned}
$$


(3) $\lambda\left(\mathrm{G}_{1} \oplus \mathrm{G}_{2}\right)=\lambda \mathrm{G}_{1} \oplus \lambda \mathrm{G}_{2}$, where $\lambda>0$

(4) $(\lambda+\mu) \mathrm{G}_{1}=\lambda \mathrm{G}_{1} \oplus \mu \mathrm{G}_{1}$, where $\lambda, \mu>0$

(5) $\left(\mathrm{G}_{1} \otimes \mathrm{G}_{2}\right)^{\lambda}=\mathrm{G}_{1}{ }^{\lambda} \otimes \mathrm{G}_{2}{ }^{\lambda}$, where $\lambda>0$

(6) $\mathrm{G}_{1}^{\lambda} \otimes \mathrm{G}_{1}{ }^{\mu}=\mathrm{G}_{1}{ }^{\lambda+\mu}$, where $\lambda, \mu>0$

Definition 2.11 (Xu, 2010) The Hamming distance of any two IVIFVs $g_{1}=\left\{\left[\alpha_{1}, \beta_{1}\right],\left[\gamma_{1}, \tau_{1}\right]\right\} g_{2}=\left\{\left[\alpha_{2}, \beta_{2}\right],\left[\gamma_{2}, \tau_{2}\right]\right\}$ is defined as follows:

$$
\mathrm{d}\left(\mathrm{g}_{1}, \mathrm{~g}_{2}\right)=\frac{1}{4}\left(\left|\alpha_{1}-\alpha_{2}\right|+\left|\beta_{1}-\beta_{2}\right|+\left|\gamma_{2}-\mathrm{c}_{2}\right|+\left|\tau_{1}-\tau_{2}\right|\right)
$$

\subsection{MM operator and PMM operator}

Definition 2.12 (Yager \& Ronald, 2001) The power average (PA) operator of the evaluation value set $G=g_{k}(k=$ $1,2, \ldots, s)$ is defined as follows:

$$
P A\left(g_{1}, g_{2}, \ldots, g_{s}\right)=\frac{\sum_{k=1}^{s}\left(1+T\left(g_{k}\right)\right) g_{k}}{\sum_{k=1}^{s}\left(1+T\left(g_{k}\right)\right)}
$$

where $T\left(g_{\mathrm{k}}\right)=\sum_{\mathrm{k}=1 \mathrm{k} \neq \mathrm{j}}^{\mathrm{s}} \operatorname{Sup}\left(g_{i}, g_{j}\right)$ indicates the degree of support of all evaluation values to $g_{i}, \operatorname{Sup}\left(g_{\mathrm{j}}, g_{\mathrm{k}}\right)=1-$ $d\left(g_{\mathrm{j}}, g_{\mathrm{k}}\right)$ and satisfiesSup $\left(g_{i}, g_{j}\right) \in[0,1]$ and $\operatorname{Sup}\left(g_{i}, g_{j}\right)=\operatorname{Sup}\left(g_{\mathrm{j}}, g_{\mathrm{i}}\right)$.

Definition 2.13 (Muirhead, 1902) The definition of the Muirhead mean (MM) operator with a positive number set $G=$ $\mathrm{g}_{\mathrm{i}}(\mathrm{i}=1,2, \ldots, \mathrm{n})$ and a parameter set $\mathrm{P}=\left(\mathrm{p}_{1}, \mathrm{p}_{2}, \ldots, \mathrm{p}_{\mathrm{n}}\right)$ is as follows:

$$
M M^{P}\left(g_{1}, g_{2}, \ldots, g_{n}\right)=\left(\frac{1}{n !} \sum_{\zeta \in T_{n}} \prod_{i=1}^{n} g_{\zeta(i)} P_{i}\right)^{\frac{1}{\sum_{i=1}^{n} p_{i}}}
$$

where $\zeta(\mathrm{i})(\mathrm{i}=1,2,3, \ldots, \mathrm{n})$ belongs to $T_{n}$, and $T_{n}$ represents any permutation of $(1,2, \ldots, \mathrm{n})$.

Definition 2.14 (Xu, Shang, Wang \& Li, 2019) The definition of the power Muirhead mean (PMM) operator of IVIFVs based on a positive number set $\mathrm{G}=\mathrm{g}_{\mathrm{i}}(\mathrm{i}=1,2, \ldots, \mathrm{n})$ and a parameter set $P=\left(p_{1}, p_{2}, \ldots, p_{n}\right)$ is as follows:

$$
P M M^{P}\left(g_{1}, g_{2}, \ldots, g_{n}\right)=\left(\frac{1}{n} \sum_{\zeta \in T} \prod_{k=1}^{n}\left(n \frac{\left(1+T\left(g_{\zeta(k)}\right)\right)}{\sum_{k=1}^{n}\left(1+T\left(g_{k}\right)\right)} g_{\zeta(k)}\right)^{p_{k}}\right)^{\frac{1}{\sum_{k=1}^{n} p_{k}}}
$$

Where $T\left(g_{\mathrm{k}}\right)=\sum_{\mathrm{k}=1 \mathrm{k} \neq \mathrm{j}}^{\mathrm{s}} \operatorname{Sup}\left(g_{i}, g_{j}\right)$ indicates the degree of support of all evaluation values to $g_{i}, \operatorname{Sup}\left(g_{\mathrm{j}}, g_{\mathrm{k}}\right)=1-$ $d\left(g_{\mathrm{j}}, g_{\mathrm{k}}\right)$ and satisfies $\operatorname{Sup}\left(g_{i}, g_{j}\right) \in[0,1]$ and $\operatorname{Sup}\left(g_{i}, g_{j}\right)=\operatorname{Sup}\left(g_{\mathrm{j}}, g_{\mathrm{i}}\right)$.

\section{IVIFPMM operators in the framework of DST}

\subsection{IVIFPMM $M_{D S T}$ operator}

The IFIVPMM operator under the DST framework is defined as follow:

Definition 3.1 A finite BI set $\widetilde{G}=\left\{\widetilde{g}_{i} \mid \widetilde{g}_{i}=\left\{\left[u_{i}^{L}, 1-v_{i}^{U}\right],\left[u_{i}^{U}, 1-v_{i}^{L}\right]\right\}, i=1,2, \ldots, n\right\}$ is transformed by IVIFS G $=$ $\left\{g_{i} \mid g_{i}=\left\{\left[u_{i}^{L}, 1-v_{i}^{U}\right],\left[u_{i}^{U}, 1-v_{i}^{L}\right]\right\}, i=1,2, \ldots, n\right\}$. Let $\left\{\left[u_{i}^{L}, 1-v_{i}^{U}\right],\left[u_{i}^{U}, 1-v_{i}^{L}\right]\right\}=\left\{\left[\operatorname{Bel}_{i}^{L}, P l_{i}^{L}\right],\left[\operatorname{Bel}_{i}^{U}, P l_{i}^{U}\right]\right\}, \quad(i=$ $1,2, \ldots, n)$ for convenience. Then the IVIFPMM $M_{D S T}$ operator is defined as follows: 
$\operatorname{IVIFPMM}{ }_{D S T}^{S}\left(\widetilde{g}_{1}, \widetilde{g}_{2}, \ldots, \widetilde{g}_{n}\right)=\left(\bigoplus_{\xi \in T_{n} B N P} \bigotimes_{k=1}^{n}\left(n \omega_{\xi N(k)} \widetilde{g}_{\xi(k)}\right)^{s_{k}}\right)^{\frac{1}{\sum_{k=1}^{n} s_{k}}}$,

where $\omega_{i}=\left(1+T\left(\widetilde{g}_{i}\right)\right) / \sum_{i=1}^{n}\left(1+T\left(\widetilde{g}_{i}\right)\right), \mathrm{T}_{\mathrm{n}} \quad$ is the full array of $(1, \quad 2, \quad \ldots, \quad \mathrm{n}), \quad$ and $T\left(\widetilde{g}_{\mathrm{j}}\right)=$ $\sum_{\mathrm{k}=1 \mathrm{k} \neq \mathrm{j}}^{n} \operatorname{Sup}\left(\widetilde{g}_{\mathrm{j}}, \widetilde{g}_{\mathrm{k}}\right), \operatorname{Sup}\left(\widetilde{g}_{\mathrm{j}}, \widetilde{g}_{\mathrm{k}}\right)=1-d\left(\widetilde{g}_{\mathrm{j}}, \widetilde{g}_{\mathrm{k}}\right)$. For the detailed definition of $\operatorname{Sup}\left(\widetilde{g}_{\mathrm{j}}, \widetilde{g}_{\mathrm{k}}\right)$ and the properties of $\operatorname{Sup}\left(\widetilde{g}_{\mathrm{j}}, \widetilde{g}_{\mathrm{k}}\right)$, see Definition 2.12 .

Theorem 3.1 (Idempotence) Based on Definition 3.1, the value aggregated by the IVIFPMM shown below:

$$
\begin{aligned}
& \operatorname{IVIFPMM} M_{D S T}^{S}\left(\widetilde{g}_{1}, \widetilde{g}_{2}, \ldots, \widetilde{g}_{n}\right)= \\
& /\left[\left[\frac{1}{n !} \sum_{\xi \in T_{n}} \prod_{k=1}^{n}\left(n \omega_{\xi(k)} \operatorname{Bel}_{\xi(k)}^{L}\right)^{s_{j}}\right]^{\frac{1}{\sum_{k=1}^{n} s_{k}}},\left[\frac{1}{n !} \sum_{\xi \in T_{n}} \prod_{k=1}^{n}\left(n \omega_{\xi(k)} P l_{\xi(k)}^{L}\right)^{s_{k}}\right]^{\frac{1}{\sum_{k=1}^{n} s_{k}}}\right], \\
& \left.\left[\left[\frac{1}{n !} \sum_{\xi \in T_{n}} \prod_{k=1}^{n}\left(n \omega_{\xi(k)} B e l_{\xi(k)}^{U}\right)^{s_{k}}\right]^{\frac{1}{\sum_{k=1}^{n} s_{k}}},\left[\frac{1}{n !} \sum_{\xi \in T_{n}} \prod_{k=1}^{n}\left(n \omega_{\xi(k)} P l_{\xi(k)}^{U}\right)^{s_{k}}\right]^{\frac{1}{\sum_{k=1}^{n} s_{k}}}\right]\right\rangle
\end{aligned}
$$

(Proof) Based on Eqs. (24)-(30) we can get $\left(\mathrm{n} \omega_{\mathrm{i}} \widetilde{g}_{i}\right)^{s}=\left\{\left[\left(\mathrm{n} \omega_{\mathrm{i}} B e l_{i}^{L}\right)^{s},\left(\mathrm{n} \omega_{\mathrm{i}} P l_{i}^{L}\right)^{s}\right],\left[\left(\mathrm{n} \omega_{\mathrm{i}} B e l_{i}^{U}\right)^{s},\left(\mathrm{n} \omega_{\mathrm{i}} P l_{i}^{U}\right)^{s}\right]\right\}$ and $\left(\mathrm{n} \omega_{\mathrm{i}} \widetilde{g}_{i}\right) \otimes\left(\mathrm{n} \omega_{\mathrm{j}} \widetilde{g}_{j}\right)=\left\{\left[\mathrm{n} \omega_{\mathrm{i}} B e l_{i}^{L} \times \mathrm{n} \omega_{\mathrm{j}} B e l_{j}^{L}, \mathrm{n} \omega_{\mathrm{i}} P l_{i}^{L} \times \mathrm{n} \omega_{\mathrm{j}} P l_{j}^{L}\right],\left[\mathrm{n} \omega_{\mathrm{i}} B e l_{i}^{U} \times \mathrm{n} \omega_{\mathrm{j}} B e l_{j}^{U}, \mathrm{n} \omega_{\mathrm{i}} P l_{i}^{U} \times \mathrm{n} \omega_{\mathrm{j}} P l_{j}^{U}\right]\right\} . T h e$ equation can be derived as follows:

$$
\begin{aligned}
& \operatorname{IVIFPMM} M_{D S T}^{S}\left(\widetilde{g}_{1}, \widetilde{g}_{2}, \ldots, \widetilde{g}_{n}\right)==\left\langle\left[\left[\frac{1}{n !} \sum_{\xi \in T_{n}} \prod_{j=1}^{n}\left(n \omega_{\xi(j)} B e l_{\xi(j)}^{L}\right)^{s_{j}}\right] \sum_{j=1}^{\frac{1}{n} s_{j}},\left[\frac{1}{n !} \sum_{\xi \in T_{n}} \prod_{j=1}^{n}\left(n \omega_{\xi(j)} P l_{\xi(j)}^{L}\right)^{s_{j}}\right]^{\frac{1}{\sum_{j=1}^{n} s_{j}}}\right],\right. \\
& \left.\left[\left[\frac{1}{n !} \sum_{\xi \in T_{n}} \prod_{j=1}^{n}\left(n \omega_{\xi(j)} B e l_{\xi(j)}^{U}\right)^{s_{j}}\right]^{\frac{1}{\sum_{j=1}^{n} s_{j}}},\left[\frac{1}{n !} \sum_{\xi \in T_{n}} \prod_{j=1}^{n}\left(n \omega_{\xi(j)} P l_{\xi(j)}^{U}\right)^{s_{j}}\right]^{\frac{1}{\sum_{j=1}^{n} s_{j}}}\right]\right\rangle
\end{aligned}
$$

Theorem 3.2 (Commutativity) Suppose $\left(\widetilde{\mathrm{g}_{1}^{\prime}}, \widetilde{\mathrm{g}}_{2}^{\prime}, \ldots, \widetilde{\mathrm{g}_{n}^{\prime}}\right)$ is a form of reorganization of $\widetilde{g}_{i}=$ $\left\{\left[\operatorname{Bel}_{i}^{L}, P l_{i}^{L}\right],\left[\operatorname{Bel}_{i}^{U}, P l_{i}^{U}\right]\right\}(i=1,2, \ldots, n)$. Then $\operatorname{IVIFPMM}_{D S T}^{S}\left(\widetilde{g_{1}}, \widetilde{g_{2}}, \ldots, \widetilde{g_{n}}\right)=\operatorname{IVIFPM} M_{D S T}^{S}\left(\widetilde{g_{1}}, \widetilde{g_{2}^{\prime}}, \ldots, \widetilde{g_{n}^{\prime}}\right)$.

(Proof) Based on the definition of IVIFVPMM $M_{D S T}$ and relationship between $\left(\widetilde{g_{1}^{\prime}}, \widetilde{g_{2}^{\prime}}, \ldots, \widetilde{g_{n}^{\prime}}\right)$ and $\left(\widetilde{g_{1}}, \widetilde{g_{2}}, \ldots, \widetilde{g_{n}}\right)$, it is easy to obtain the following expression:

$$
\begin{aligned}
& \operatorname{IVIFPMM} \underset{D S T}{S}\left(\widetilde{g}_{1}, \widetilde{g}_{2}, \ldots, \widetilde{g}_{n}\right)=\left\langle\left[\left[\frac{1}{n !} \sum_{\xi \in T_{n}} \prod_{j=1}^{n}\left(n \omega_{\xi(j)} B e l_{\xi(j)}^{L}\right)^{s_{j}}\right] \sum_{j=1}^{\frac{1}{n} s_{j}},\left[\frac{1}{n !} \sum_{\xi \in T_{n}} \prod_{j=1}^{n}\left(n \omega_{\xi(j)} P l_{\xi(j)}^{L}\right)^{s_{j}}\right] \sum_{j=1}^{\frac{1}{n} s_{j}}\right],\right. \\
& \left.\left[\left[\frac{1}{n !} \sum_{\xi \in T_{n}} \prod_{j=1}^{n}\left(n \omega_{\xi(j)} B e l_{\xi(j)}^{U}\right)^{s_{j}}\right]^{\frac{1}{\sum_{j=1}^{n} s_{j}}},\left[\frac{1}{n !} \sum_{\xi \in T_{n}} \prod_{j=1}^{n}\left(n \omega_{\xi(j)} P l_{\xi(j)}^{U}\right)^{s_{j}}\right]^{\frac{1}{\sum_{j=1}^{n} s_{j}}}\right]\right\rangle \\
& =\left[\left[\frac{1}{n !} \sum_{\xi \in T_{n}^{\prime}} \prod_{j=1}^{n}\left(n \omega_{\xi(j)} B e l_{\xi(j)}^{L}\right)^{s_{j}}\right]^{\frac{1}{n_{j=1}^{n}} s_{j}},\left[\frac{1}{n !} \sum_{\xi \in T_{n}^{\prime}} \prod_{j=1}^{n}\left(n \omega_{\xi(j)} P l_{\xi(j)}^{L}\right)^{s_{j}}\right]^{\frac{1}{n} s_{j}} s_{j}\right] \text {, }
\end{aligned}
$$




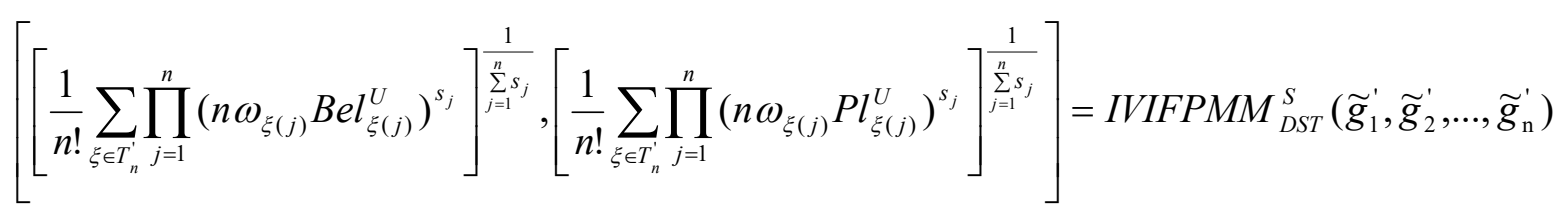

Theorem 3.3 (Boundedness). If $\tilde{\mathrm{g}}^{+}=\max _{i=1}^{n}\left\{\left[\mathrm{Bel}_{i}^{L}, P l_{i}^{L}\right],\left[\operatorname{Bel}_{i}^{U}, P l_{i}^{U}\right]\right\}$ and $\tilde{\mathrm{g}}^{-}=\min _{i=1}^{n}\left\{\left[B e l_{i}^{L}, P l_{i}^{L}\right],\left[\operatorname{Bel}_{i}^{U}, P l_{i}^{U}\right]\right\}$, then $\widetilde{\mathrm{g}}^{-}<\operatorname{IVIFPMM} M_{D S T}^{S}\left(\widetilde{g_{1}}, \widetilde{g_{2}}, \ldots, \widetilde{g_{n}}\right)<\widetilde{\mathrm{g}}^{+}$.

(Proof) From Definition 2.10, we can easily obtain that:

$n \omega_{\xi(j)} \widetilde{g}_{\xi(j)} \leq n \omega_{\xi(j)} \widetilde{g}_{\xi(j)}^{+}$and $\left(n \omega_{\xi(j)} \widetilde{g}_{\xi(j)}\right)^{s_{j}} \leq\left(n \omega_{\xi(j)} \widetilde{g}_{\xi(j)}^{+}\right)^{s_{j}}$,

Therefore $\bigotimes_{j=1}^{n}\left(n \omega_{\xi N P} \widetilde{g}_{\xi(j)}\right)^{s_{j}} \leq \bigotimes_{j=1}^{n}\left(n \omega_{\xi N P} \widetilde{g}^{+}\right)^{s_{j}}$

Further $\bigoplus_{\xi \in T_{n}} \bigotimes_{B N P}^{n}\left(n \omega_{\xi(j)} \widetilde{g}_{\xi(j)}\right)^{s_{j}} \leq \bigoplus_{\xi \in T_{n}} \bigotimes_{B N P}^{n}\left(n \omega_{\xi=1} \tilde{g}_{B N P} \tilde{g}^{+}\right)^{s_{j}}$

thus $\operatorname{IVIFPMM} M_{D S T}^{S}\left(\widetilde{g}_{1}^{\prime}, \widetilde{g}_{2}^{\prime}, \ldots, \widetilde{g}_{\mathrm{n}}^{\prime}\right)=\left(\bigoplus_{\xi \in T_{n}} \bigotimes_{B N P}^{n}\left(n \omega_{\xi(j)} \widetilde{g}_{\xi(j)}\right)^{s_{j}}\right)^{\sum^{\frac{1}{n} s_{j}} s_{j}}$

$$
\leq\left(\bigoplus_{\xi \in T_{n}} \bigotimes_{B N P}^{n}\left(n \omega_{\xi(j)} \widetilde{g}^{+}\right)^{s_{j}}\right)^{\frac{1}{\sum_{j=1}^{n} s_{j}}}=\widetilde{g}^{+} .
$$

Therefore, it can be obtained that:

$$
\widetilde{\mathrm{g}}^{-}<\operatorname{IVIFPM} M_{D S T}^{S}\left(\widetilde{\mathrm{g}_{1}}, \widetilde{\mathrm{g}_{2}}, \ldots, \widetilde{\mathrm{g}_{n}}\right)<\widetilde{\mathrm{g}}^{+}
$$

\subsection{IVIFWPMM $M_{D S T}$ operator}

The IVIFPM $M_{D S T}$ operator calculates the average power between any number of criteria but does not consider the weights of the criteria. The IVIFWPMM operator under the DST framework which contains the weight of the criteria is defined as follow:

Definition 3.2. A finite BI set $\widetilde{G}=\left\{\widetilde{g}_{i} \mid \widetilde{g}_{i}=\left\{\left[u_{i}^{L}, 1-v_{i}^{U}\right],\left[u_{i}^{U}, 1-v_{i}^{L}\right]\right\}, i=1,2, \ldots, n\right\}$ is transformed by IVIFVs $\mathrm{G}=$ $\left\{g_{i} \mid g_{i}=\left\{\left[u_{i}^{L}, 1-v_{i}^{U}\right],\left[u_{i}^{U}, 1-v_{i}^{L}\right]\right\}, i=1,2, \ldots, n\right\}$. Let $\left\{\left[u_{i}^{L}, 1-v_{i}^{U}\right],\left[u_{i}^{U}, 1-v_{i}^{L}\right]\right\}=\left\{\left[\operatorname{Bel}_{i}^{L}, P l_{i}^{L}\right],\left[\operatorname{Bel}_{i}^{U}, P l_{i}^{U}\right]\right\}, \quad(i=$ $1,2, \ldots, n) \cdot w_{i}(i=1,2, \ldots, n)$ is the weight corresponding to $g_{i}(i=1,2,3 \ldots, n)$, where $\sum_{j=1}^{n} \omega_{j}=1$ and $0 \leq \omega_{j} \leq 1$. The IVIFWPMM $M_{D S T}$ operator of $\left(\widetilde{\mathrm{g}_{1}}, \widetilde{\mathrm{g}_{2}}, \ldots, \widetilde{\mathrm{g}_{\mathrm{n}}}\right)$ is defined as follow:

$$
\operatorname{IVIFWPMM} M_{D S T}^{S}\left(\widetilde{g}_{1}, \widetilde{g}_{2}, \ldots, \widetilde{g}_{n}\right)=\left(\bigoplus_{\xi \in T_{n}} \bigotimes_{B N P}^{\mathrm{n}}\left(n \frac{\omega_{\xi(j)}\left(1+T\left(\widetilde{g}_{\xi N P}\right)\right)}{\sum_{j=1}^{n} \omega_{j}\left(1+T\left(\widetilde{g}_{j}\right)\right)} \widetilde{g}_{\xi(j)}\right)^{s_{j}}\right)^{\frac{1}{\sum_{j=1}^{n} s_{j}}}
$$

where $\mathrm{T}\left(\widetilde{g_{\tau}}\right)=\sum_{\mu=1, \mu \neq \tau}^{\mathrm{n}} \operatorname{Sup}\left(\widetilde{g_{\tau}}, \widetilde{g_{\mu}}\right), \operatorname{Sup}\left(\widetilde{g_{\tau}}, \widetilde{g_{\mu}}\right)=1-\mathrm{d}\left(\widetilde{g_{\tau}}, \widetilde{g_{\mu}}\right)$ and $\operatorname{Sup}\left(\widetilde{g_{\tau}}, \widetilde{g_{\mu}}\right)$ denotes the support degree for $\widetilde{g_{\mu}}$ from $\widetilde{g_{\tau}}$.

To simplify the above operator, let $\delta_{j}=\omega_{\xi(j)}\left(1+T\left(\widetilde{\xi_{(j)}}\right)\right) / \sum_{j=1}^{n} \omega_{j}\left(1+T\left(\widetilde{g_{j}}\right)\right)$, then the above equation can be expressed as : 


$$
\text { IVIFWPMM } M_{D S T}^{S}\left(\widetilde{g}_{1}, \widetilde{g}_{2}, \ldots, \widetilde{g}_{n}\right)=\left(\bigoplus_{\xi \in T_{n}} \bigotimes_{B N P}^{\mathrm{n}}\left(n \delta_{\xi=1} \widetilde{g}_{\xi N(j)}\right)^{s_{j}}\right)^{\frac{1}{\sum_{j=1}^{n} s_{j}}}
$$

Theorem 3.4 (Idempotence) Based on Definition 3.2, the value aggregated by the IVIFWPMM $M_{D S T}$ operator is still a BI, as shown below:

$$
\begin{gathered}
\operatorname{IVIFWPMM} M_{D S T}^{S}\left(\widetilde{g}_{1}, \widetilde{g}_{2}, \ldots, \widetilde{g}_{n}\right)=\left\langle\left[\left[\frac{1}{n !} \sum_{\xi \in T_{n}} \prod_{j=1}^{n}\left(n \delta_{\xi(j)} B e l_{\xi(j)}^{L}\right)^{s_{j}}\right]^{\frac{1}{n_{j=1}^{n} s_{j}}},\left[\frac{1}{n !} \sum_{\xi \in T_{n}} \prod_{j=1}^{n}\left(n \delta_{\xi(j)} P l_{\xi(j)}^{L}\right)^{s_{j}}\right] \sum_{j=1}^{\frac{1}{n} s_{j}}\right.\right. \\
\left.\left.\qquad\left[\left[\frac{1}{n !} \sum_{\xi \in T_{n}} \prod_{j=1}^{n}\left(n \delta_{\xi(j)} B e l_{\xi(j)}^{U}\right)^{s_{j}}\right]^{\frac{1}{\sum_{j=1}^{n} s_{j}}},\left[\frac{1}{n !} \sum_{\xi \in T_{n}} \prod_{j=1}^{n}\left(n \delta_{\xi(j)} P l_{\xi(j)}^{U}\right)^{s_{j}}\right]\right]_{j=1}^{\frac{1}{\sum_{j}^{n} s_{j}}}\right]\right\rangle
\end{gathered}
$$

(Proof) The proof here is similar to that of Eq. (47). It is not repeated here.

Theorem 3.5 (Commutativity) Suppose $\left(\widetilde{\mathrm{g}_{1}^{\prime}}, \widetilde{\mathrm{g}}_{2}^{\prime}, \ldots, \widetilde{\mathrm{g}}_{n}^{\prime}\right)$ is a form of reorganization of $\widetilde{g}_{i}=$ $\left\{\left[B e l_{i}^{L}, P l_{i}^{L}\right],\left[B e l_{i}^{U}, P l_{i}^{U}\right]\right\}(i=1,2, \ldots, n)$, then IVIFWPMM $M_{D S T}^{S}\left(\widetilde{g_{1}}, \widetilde{g_{2}}, \ldots, \widetilde{g_{n}}\right)=I V I F W P M M_{D S T}^{S}\left(\widetilde{g_{1}^{\prime}}, \widetilde{g_{2}^{\prime}}, \ldots, \widetilde{g_{n}^{\prime}}\right)$.

(Proof) The proof here is similar to that of Theorem 3.2. It is not repeated here.

Theorem 3.6 (Boundedness) If $\tilde{\mathrm{g}}^{+}=\max _{i=1}^{n}\left\{\left[\operatorname{Bel}_{i}^{L}, P l_{i}^{L}\right],\left[\operatorname{Bel}_{i}^{U}, P l_{i}^{U}\right]\right\}$ and $\tilde{\mathrm{g}}^{-}=\min _{i=1}^{n}\left\{\left[\operatorname{Bel}_{i}^{L}, P l_{i}^{L}\right],\left[\operatorname{Bel}_{i}^{U}, P l_{i}^{U}\right]\right\}$, then $\tilde{\mathrm{g}}^{-}<I V I F W P M M_{D S T}^{S}\left(\widetilde{g_{1}}, \widetilde{g_{2}}, \ldots, \widetilde{g_{n}}\right)<\widetilde{\mathrm{g}}^{+}$.

(Proof) The proof here is similar to that of Theorem 3.3.It is not repeated here.

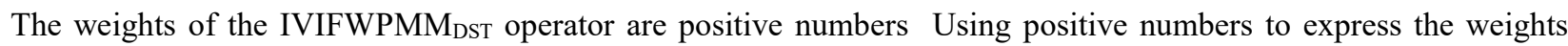
sometimes cannot accurately express the decision maker's preference. Therefore, it is necessary to define a $I V I F \bar{W} P M M_{D S T}$ operator, where the weights are IVIFVs, as shown follows.

Definition 3.3 The finite [BI] set $\widetilde{G}=\left\{\widetilde{g}_{i} \mid \widetilde{g}_{i}=\left\{\left[u_{i}^{L}, 1-v_{i}^{U}\right],\left[u_{i}^{U}, 1-v_{i}^{L}\right]\right\}, i=1,2, \ldots, n\right\}$ is transformed by IVIFS $\mathrm{G}=\left\{g_{i} \mid g_{i}=\left\{\left[u_{i}^{L}, 1-v_{i}^{U}\right],\left[u_{i}^{U}, 1-v_{i}^{L}\right]\right\}, i=1,2, \ldots, n\right\} . \operatorname{Let}\left\{\left[u_{i}^{L}, 1-v_{i}^{U}\right],\left[u_{i}^{U}, 1-v_{i}^{L}\right]\right\}=\left\{\left[\operatorname{Bel}_{i}^{L}, P l_{i}^{L}\right],\left[\operatorname{Bel}_{i}^{U}, P l_{i}^{U}\right]\right\}$,

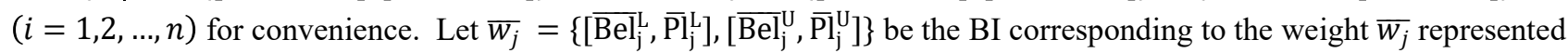
by the IVIFV. When $\overline{\mathrm{W}}=\left(\overline{w_{1}}, \overline{w_{2}}, \ldots, \overline{w_{n}}\right)$ satisfies the following two conditions, $\mathrm{W}$ is a normalized IVIFN vector (1) There is at least one normalized vector $a \in I$, where $I=\left\{a=\left(a_{1}, a_{2}, \ldots, a_{z}\right) \mid \overline{\mathrm{Bel}}_{\mathrm{j}}^{\mathrm{L}} \leq a_{j} \leq \overline{\mathrm{Pl}}_{\mathrm{j}}^{\mathrm{U}}, j=1,2, \ldots, z\right\}, \sum_{j=1}^{z} a_{j}=$ $1\}$ is a set of normalized weight vectors; (2) $\overline{\mathrm{Bel}}_{\mathrm{j}}^{\mathrm{L}}$ and $\overline{\mathrm{Pl}}_{\mathrm{j}}^{\mathrm{U}},(j=1,2, \ldots, z)$ are all attainable in I.

The IVIF $\bar{W} P M M_{D S T}$ operator of $\left(\widetilde{\mathrm{g}_{1}}, \widetilde{\mathrm{g}_{2}}, \ldots, \widetilde{\mathrm{g}_{\mathrm{n}}}\right)$ is define as follow:

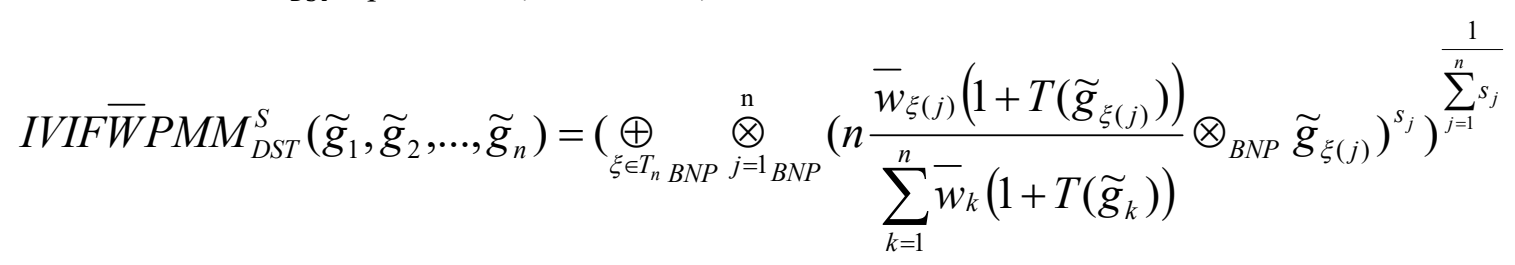

where $\mathrm{T}\left(\widetilde{g_{\tau}}\right)=\sum_{\mu=1, \mu \neq \tau}^{\mathrm{n}} \operatorname{Sup}\left(\widetilde{g_{\tau}}, \widetilde{g_{\mu}}\right), \operatorname{Sup}\left(\widetilde{g_{\tau}}, \widetilde{g_{\mu}}\right)=1-\mathrm{d}\left(\widetilde{g_{\tau}}, \widetilde{g_{\mu}}\right)$ and $\operatorname{Sup}\left(\widetilde{g_{\tau}}, \widetilde{g_{\mu}}\right)$ denotes the support degree for $\widetilde{g_{\mu}}$ from $\widetilde{g_{\tau}}$.

To simplify the above operator, let $\bar{\delta}_{j}=\bar{\omega}_{\xi(j)}\left(1+T\left(\widetilde{\xi_{(j)}}\right)\right) / \sum_{j=1}^{n} \bar{\omega}_{j}\left(1+T\left(\widetilde{g_{j}}\right)\right)$, then the above equation can be expressed as the following form:

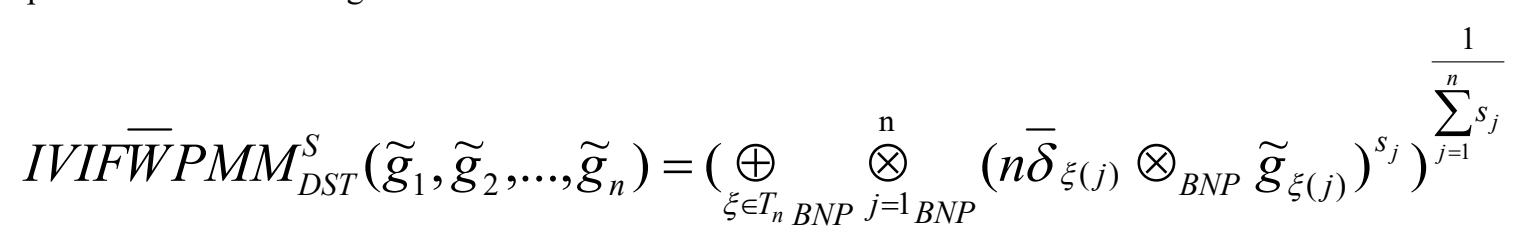


Theorem 3.7 (Idempotence) Based on Definition 3.3, the value aggregated by the IVIFVW $\bar{P} M M_{\text {DST }}$ operator is still [BI],as shown below:

$$
\begin{aligned}
& \operatorname{IVIF} \bar{W} P M M_{D S T}^{S}\left(\widetilde{g}_{1}, \widetilde{g}_{2}, \ldots, \widetilde{g}_{n}\right)=
\end{aligned}
$$

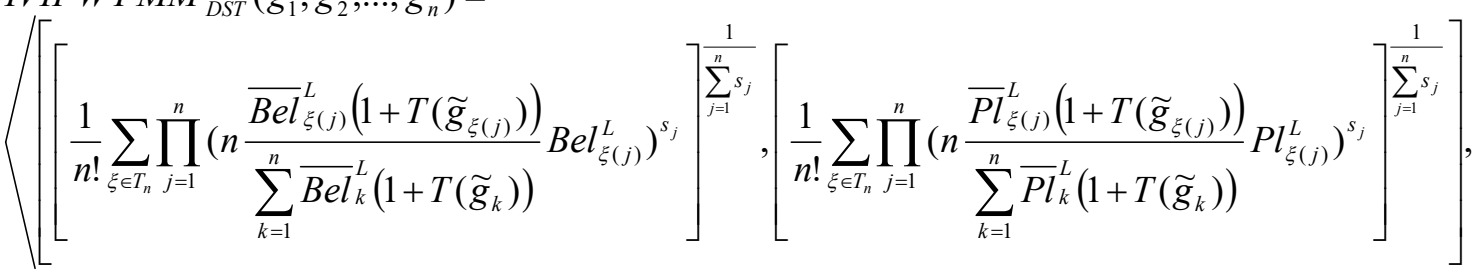

$$
\begin{aligned}
& \left.\left[\left[\frac{1}{n !} \sum_{\xi \in T_{n}} \prod_{j=1}^{n}\left(n \frac{\overline{B e l}_{\xi(j)}^{U}\left(1+T\left(\widetilde{g}_{\xi(j)}\right)\right)}{\sum_{k=1}^{n} \overline{B e l}_{k}^{U}\left(1+T\left(\widetilde{g}_{k}\right)\right)} B e l_{\xi(j)}^{U}\right)^{s_{j}}\right]^{\frac{1}{\sum_{j=1}^{n} s_{j}}},\left[\frac{1}{n !} \sum_{\xi \in T_{n}} \prod_{j=1}^{n}\left(n \frac{\overline{P l} l_{\xi(j)}^{U}\left(1+T\left(\widetilde{g}_{\xi(j)}\right)\right)}{\sum_{k=1}^{n} \overline{P l}_{k}^{U}\left(1+T\left(\widetilde{g}_{k}\right)\right)} P l_{\xi(j)}^{U}\right)^{s_{j}}\right]^{\frac{1}{\sum_{j=1}^{n} s_{j}}}\right]\right\rangle
\end{aligned}
$$

(Proof) The proof here is similar to that of Theorem 3.1, so it will not be repeated here.

Theorem 3.8.(Commutativity) Suppose $\left(\widetilde{\mathrm{g}}_{1}, \widetilde{\mathrm{g}}_{2}, \ldots, \widetilde{\mathrm{g}_{n}^{\prime}}\right)$ is a form of reorganization of $\widetilde{g}_{i}=$ $\left\{\left[B e l_{i}^{L}, P l_{i}^{L}\right],\left[B e l_{i}^{U}, P l_{i}^{U}\right]\right\}(i=1,2, \ldots, n)$, then $\operatorname{IVIF} \bar{W} P M M_{D S T}^{S}\left(\widetilde{g_{1}}, \widetilde{g_{2}}, \ldots, \widetilde{g_{n}}\right)=\operatorname{IVIF} \bar{W} P M M_{D S T}^{S}\left(\widetilde{g_{1}^{\prime}}, \widetilde{g_{2}^{\prime}}, \ldots, \widetilde{g_{n}^{\prime}}\right)$.

(Proof) The proof here is similar to that of Theorem 3.2, so it will not be repeated here.

Theorem 3.9.(Boundedness). If $\tilde{\mathrm{g}}^{+}=\max _{i=1}^{n}\left\{\left[\operatorname{Bel}_{i}^{L}, P l_{i}^{L}\right],\left[\operatorname{Bel}_{i}^{U}, P l_{i}^{U}\right]\right\}$ and $\tilde{\mathrm{g}}^{-}=\min _{i=1}^{n}\left\{\left[B e l_{i}^{L}, P l_{i}^{L}\right],\left[B e l_{i}^{U}, P l_{i}^{U}\right]\right\}$, then $\widetilde{\mathrm{g}}^{-}<I V I F \bar{W} P M M_{D S T}^{S}\left(\widetilde{g_{1}}, \widetilde{g_{2}}, \ldots, \widetilde{g_{n}}\right)<\widetilde{\mathrm{g}}^{+}$.

(Proof) The proof here is similar to that of Theorem 3.3, so it will not be repeated here.

\section{MCDM method with IVIFVs in the framework of DST}

Considering the following MCDM problem: there are $q$ local criteria, denoted by $R=\left\{r_{1}, r_{2}, \ldots, r_{q}\right\}$ and $\mathrm{p}$ alternatives, denoted by $T=\left\{t_{1}, t_{2}, \ldots, t_{p}\right\}$. If the weights of criteria are positive numbers such that $w_{l}>0(l=$ $1,2, \ldots, q)$ and $\sum_{l=1}^{q} w_{l}=1$. Else, if criteria weights are IVIFNs, then weight vector is denoted by an interval intuitive weight vector $\bar{w}=\left(\overline{w_{1}}, \overline{w_{2}}, \ldots, \overline{w_{q}}\right)$ with $\overline{w_{l}}=\left\{\left[\overline{\operatorname{Bel}}_{1}^{\mathrm{L}}, \overline{\mathrm{Pl}}_{1}^{\mathrm{L}}\right],\left[\overline{\mathrm{Bel}_{1}^{U}}, \overline{\mathrm{Pl}_{1}^{\mathrm{U}}}\right]\right\},(l=1,2, \ldots, q)$. The aim of the MCDM method is to sort the columns of a decision matrix $B=\left(b_{j k}\right)_{p \times q}$, where $b_{j k}$ denotes the IVIFV preference value of the alternative $t_{j}$ with respect to the criterion $r_{k}$.

The detailed steps of the proposed MCDM method are as follows:

Step 1 Normalize the decision matrix $B=\left(b_{j k}\right)_{p \times q}$. The higher the value of $b_{j k}$, the higher the positive preference.

Step 2 Convert IVIFV $b_{j k}$ to BI $\widetilde{h_{\mathrm{jk}}}$ by Eqs. (22) - (24).

Step 3 Calculate the support: $\operatorname{Sup}\left(\widetilde{h_{\mathrm{jk}}} \widetilde{h_{j l}}\right)(j=1,2 \ldots, p ; l, k=1,2, \ldots, q ; l \neq k)$.

$$
\operatorname{Sup}\left(\widetilde{h_{j k}}, \widetilde{h_{j l}}\right)=1-d\left(\widetilde{h_{j k}}, \widetilde{h_{j l}}\right),(j=1,2, \ldots, p ; l, k=1,2, \ldots, q ; l \neq k),
$$

where $d\left(\widetilde{h_{j k}}, \widetilde{h_{j l}}\right)$ denotes the Hamming distance between $\widetilde{h_{\mathrm{jk}}}$ and $\widetilde{h_{j l}}$ in the DST framework.

Step 4 Calculate $T\left(\widetilde{h_{j k}}\right)$ of $\widetilde{h_{\mathrm{jk}}}$ by the support of other $\widetilde{h_{j l}}$.

$$
T\left(\widetilde{h_{j k}}\right)=\sum_{l=1, l \neq k}^{q} \operatorname{Sup}\left(\widetilde{h_{j k}}, \widetilde{h_{j l}}\right),(j=1,2 \ldots, p ; k=1,2, \ldots, q) .
$$

Step 5 Calculate $\delta_{l}, \bar{\delta}_{l}$. If $\bar{w}=\left\{\left[\overline{\mathrm{Bel}}_{\mathrm{l}}^{\mathrm{L}}, \overline{\mathrm{P}}_{\mathrm{l}}^{\mathrm{L}}\right],\left[\overline{\mathrm{Be}}_{\mathrm{l}}^{\mathrm{U}}, \overline{\mathrm{P}}_{\mathrm{l}}^{\mathrm{U}}\right]\right\}$ satisfies $\sum_{l=1}^{q} \overline{\mathrm{Bel}}_{\mathrm{l}}^{\mathrm{U}}+\max _{k}\left(\overline{\mathrm{P}}_{\mathrm{k}}^{\mathrm{U}}-\overline{\mathrm{Bel}}_{\mathrm{k}}^{\mathrm{L}}\right) \leq 1$ and $\sum_{l=1}^{q} \overline{\mathrm{Pl}}_{\mathrm{L}}^{\mathrm{L}}-$ $\max _{k}\left(\overline{\mathrm{Pl}}_{\mathrm{k}}^{\mathrm{U}}-\overline{\mathrm{Bel}}_{\mathrm{k}}^{\mathrm{L}}\right) \geq 1,(k=1,2, \ldots, q)$, then $\bar{w}$ is normalized. If not, $\bar{w}=\left\{\left[\overline{\mathrm{Bel}}_{1}^{\mathrm{L}}, \overline{\mathrm{Pl}}_{1}^{\mathrm{L}}\right],\left[\overline{\mathrm{Bel}}_{1}^{\mathrm{U}}, \overline{\mathrm{Pl}}_{1}^{\mathrm{U}}\right]\right\}$ is normalized by the following two formulas (Shafer \& Glenn, 1976):

$$
\begin{aligned}
& \overline{B e l}_{l}^{L}=\max \left\{\overline{B e}_{l}^{L}, 1-\sum_{l=1, l \neq k}^{q} \overline{P l}_{l}^{U}\right\} \quad 1=1,2, \ldots, q \\
& \overline{P l}_{l}^{L}=\max \left\{\overline{P l}_{l}^{L}, 1-\sum_{l=1, j \neq k}^{q} \overline{B e l}_{l}^{U}\right\}, \quad l=1,2, \ldots, q
\end{aligned}
$$




$$
\begin{aligned}
& \overline{B e}_{l}^{U}=\max \left\{\overline{B e}_{l}^{U}, 1-\sum_{l=1, l \neq k}^{q} \overline{P l}_{l}^{L}\right\}, 1=1,2, \ldots, q \\
& \overline{P l}_{l}^{U}=\max \left\{\overline{P l}_{l}^{U}, 1-\sum_{l=1, j \neq k}^{q} \overline{B e l}_{l}^{L}\right\}, \quad 1=1,2, \ldots, q
\end{aligned}
$$

and then

$$
\delta_{j k}=\frac{w_{k}\left(1+T\left(\widetilde{h_{j k}}\right)\right)}{\sum_{l=1}^{q} w_{l}\left(1+T\left(\widetilde{h_{j l}}\right)\right)} \text { or } \overline{\delta_{j k}}=\frac{\overline{w_{k}}\left(1+T\left(\widetilde{h_{j k}}\right)\right)}{\sum_{l=1}^{q} \overline{w_{l}}\left(1+T\left(\widetilde{h_{j l}}\right)\right)}
$$

Step 6 Use the $I V I F W P M M_{D S T}$ operator (i.e., $\mathrm{Eq}(50)$ ) or $I V I F \bar{W} P M M_{D S T}$ operator (i.e., $\mathrm{Eq}(51)$ ) to calculate the comprehensive value of each alternative.

Step 7 Calculate the $S F_{D S T}\left(\widetilde{h_{j}}\right)$ and $A F_{D S T}\left(\widetilde{h_{j}}\right)$ by Eqs.(34) - (35), respectively. For convenience, $S F_{D S T}\left(\widetilde{h_{j}}\right)$ and $A F_{D S T}\left(\widetilde{h_{j}}\right)$ are abbreviated as $S_{j}$ and $A_{j}$, respectively.

Step 8 Sort the alternatives by using $S_{j}$ and $A_{j},(j=1,2, \ldots, p)$ to get the optimal alternative.

\section{Application and verification}

\subsection{Application of the presented method}

The proposed method is used to solve a practical MCDM problem (Xu, Shang, Wang \& Li, 2019) that a research team optimally select smart device alternative.

Example 5.1 A university research team plans to buy a new batch of smart devices for the team. After preliminary screening, there are four alternatives $T=\left\{t_{1}, t_{2}, t_{3}, t_{4}\right\}$ to meet the requirements. To select the most suitable alternative, there are four criteria $R=\left\{r_{1}, r_{2}, r_{3}, r_{4}\right\}$ needing to be considered, i.e., equipment price $\left(r_{1}\right)$, team demand $\left(r_{2}\right)$, compatibility with the team's current smart equipment $\left(r_{3}\right)$, and after-sale service quality $\left(r_{4}\right)$. The weight vector of the criteria $r_{i}(i=1,2,3,4)$ is $w=(0.3,0.25,0.25,0.2)^{T} . P t(t=1,2,3)$ are three experts who evaluate the four programs. $\gamma=(0.32,0.45,0.23)^{T}$ is the weight vector of the decision results of the three experts. The evaluation value $\mathrm{G}_{\mathrm{k}}=g_{j k}^{l}(k, j=1,2,3,4, l=1,2,3)$ is in the form of IVIFVs. Tables 1-3 shows three decision matrices.

Table 1. The IVIF decision matrix $\mathrm{G}_{1}$ given by expert $\mathrm{P} 1$.

\begin{tabular}{ccccc}
\hline & $r_{1}$ & $r_{2}$ & $r_{3}$ & $r_{4}$ \\
\hline$t_{1}$ & $([0.5,0.6],[0.3,0.4])$ & $([0.4,0.6],[0.2,0.3])$ & $([0.6,0.8],[0.1,0.2])$ & $([0.6,0.7],[0.1,0.3])$ \\
$t_{2}$ & $([0.7,0.8],[0.1,0.2])$ & $([0.5,0.6],[0.1,0.2])$ & $([0.3,0.5],[0.3,0.4])$ & $([0.7,0.8],[0.1,0.2])\}$ \\
$t_{3}$ & $([0.4,0.6],[0.2,0.3])$ & $([0.5,0.7],[0.1,0.2])$ & $([0.4,0.6],[0.3,0.4])$ & $([0.5,0.6],[0.2,0.3])$ \\
$t_{4}$ & $([0.6,0.7],[0.1,0.3])$ & $([0.5,0.6],[0.2,0.3])$ & $([0.4,0.5],[0.2,0.4])$ & $([0.4,0.7],[0.2,0.3])$ \\
\hline
\end{tabular}

Table 2. The IVIF decision matrix $\mathrm{G}_{2}$ given by expert $\mathrm{P} 2$.

\begin{tabular}{ccccc}
\hline & $r_{1}$ & $r_{2}$ & $r_{3}$ & $r_{4}$ \\
\hline$t_{1}$ & $([0.7,0.8],[0.1,0.2])$ & $([0.5,0.6],[0.1,0.3])$ & $([0.4,0.5],[0.2,0.4])$ & $([0.5,0.8],[0.1,0.2])$ \\
$t_{2}$ & $([0.5,0.6],[0.2,0.3])$ & $([0.6,0.7],[0.2,0.3])$ & $([0.5,0.5],[0.2,0.3])$ & $([0.6,0.7],[0.1,0.2])$ \\
$t_{3}$ & $([0.4,0.5],[0.1,0.2])$ & $([0.6,0.8],[0.1,0.2])$ & $([0.5,0.7],[0.2,0.3])$ & $([0.6,0.7],[0.1,0.3])$ \\
$t_{4}$ & $([0.5,0.6],[0.2,0.3])$ & $([0.4,0.5],[0.3,0.4])$ & $([0.6,0.8],[0.1,0.2])$ & $([0.5,0.8],[0.1,0.2])$ \\
\hline
\end{tabular}

Table 3. The IVIF decision matrix $\mathrm{G}_{3}$ given by expert $\mathrm{P} 3$.

\begin{tabular}{ccccc}
\hline & $r_{1}$ & $r_{2}$ & $r_{3}$ & $r_{4}$ \\
\hline$t_{1}$ & $([0.6,0.6],[0.2,0.3])$ & $([0.5,0.8],[0.1,0.2])$ & $([0.5,0.7],[0.1,0.2])$ & $([0.6,0.7],[0.2,0.3])$ \\
$t_{2}$ & $([0.7,0.8],[0.1,0.2])$ & $([0.4,0.5],[0.3,0.4])$ & $([0.6,0.7],[0.1,0.2])$ & $([0.5,0.6],[0.2,0.3])$ \\
$t_{3}$ & $([0.6,0.6],[0.2,0.3])$ & $([0.5,0.7],[0.2,0.3])$ & $([0.6,0.8],[0.1,0.2])$ & $([0.5,0.6],[0.3,0.4])$ \\
$t_{4}$ & $([0.4,0.5],[0.3,0.4])$ & $([0.6,0.8],[0.1,0.2])$ & $([0.5,0.6],[0.2,0.3])$ & $([0.7,0.8],[0.1,0.2])$ \\
\hline
\end{tabular}

Using the proposed method, the MCDM problems can be solved via the following steps:

Step 1 Since all attributes are beneficial types, there is no need to normalize the decision matrices. 
Step 2 Convert IVIFV $g_{j k}^{l}$ to [BI] $\widetilde{g_{j k}^{l}}$. For example, all IVIFVs in Table 1 are converted into BIs, which are shown in Table 4

Table 4. The BIs decision matrix converted from Table 1.

\begin{tabular}{ccccc}
\hline & $r_{1}$ & $r_{2}$ & $r_{3}$ & $r_{4}$ \\
\hline$t_{1}$ & $([0.5,0.6],[0.6,0.7])$ & $([0.4,0.7],[0.6,0.8])$ & $([0.6,0.8],[0.8,0.9])$ & $([0.6,0.7],[0.7,0.9])$ \\
$t_{2}$ & $([0.7,0.8],[0.8,0.9])$ & $([0.5,0.8],[0.6,0.9])$ & $([0.3,0.6],[0.5,0.7])$ & $([0.7,0.8],[0.8,0.9])$ \\
$t_{3}$ & $([0.4,0.7],[0.6,0.8])$ & $([0.5,0.8],[0.7,0.9])$ & $([0.4,0.6],[0.6,0.7])$ & $([0.5,0.7],[0.6,0.8])$ \\
$t_{4}$ & $([0.6,0.7],[0.7,0.9])$ & $([0.5,0.7],[0.6,0.8])$ & $([0.4,0.6],[0.5,0.8])$ & $([0.4,0.7],[0.7,0.8])$ \\
\hline
\end{tabular}

Step 3 Calculate $\operatorname{Sup}\left(\widetilde{g_{j k}^{l}}, \widetilde{g_{j k}^{h}}\right)(j, k=1,2,3,4 ; h, l=1,2,3, h \neq l)$. Let $\operatorname{Sup}_{h}^{l}=\operatorname{Sup}\left(\widetilde{g_{j k}^{l}}, \widetilde{g_{j k}^{h}}\right)(j, k=1,2,3,4 ; h, l=$ $1,2,3, h \neq l$ ) for convenience, then

$$
\begin{aligned}
& \text { Sup }_{1}^{2}=\text { Sup }_{2}^{1}=\left[\begin{array}{llll}
0.800 & 0.950 & 0.800 & 0.925 \\
0.850 & 0.900 & 0.900 & 0.950 \\
0.925 & 0.950 & 0.900 & 0.925 \\
0.925 & 0.900 & 0.800 & 0.900
\end{array}\right], \\
& \text { Sup }_{1}^{3}=\text { Sup }_{3}^{1}=\left[\begin{array}{cccc}
0.925 & 0.875 & 0.950 & 0.975 \\
1 & 0.850 & 0.775 & 0.850 \\
0.950 & 0.950 & 0.800 & 0.950 \\
0.825 & 0.875 & 0.925 & 0.850
\end{array}\right], \\
& \text { Sup }_{3}^{2}=\text { Sup }_{2}^{3}=\left[\begin{array}{llll}
0.875 & 0.950 & 0.850 & 0.900 \\
0.850 & 0.850 & 0.875 & 0.900 \\
0.875 & 0.900 & 0.900 & 0.875 \\
0.900 & 0.775 & 0.875 & 0.950
\end{array}\right] .
\end{aligned}
$$

Step 4 Calculate $T_{l}=T\left(\widetilde{g_{j k}^{l}}\right)(l=1,2,3)$ of $\widetilde{g_{j k}^{l}}$, then

$$
\begin{aligned}
T_{1} & =\left[\begin{array}{llll}
1.725 & 1.825 & 1.750 & 1.900 \\
1.850 & 1.750 & 1.675 & 1.800 \\
1.875 & 1.900 & 1.700 & 1.875 \\
1.750 & 1.775 & 1.725 & 1.750
\end{array}\right], \\
T_{2} & =\left[\begin{array}{llll}
1.675 & 1.875 & 1.650 & 1.825 \\
1.700 & 1.750 & 1.775 & 1.850 \\
1.800 & 1.850 & 1.800 & 1.800 \\
1.825 & 1.675 & 1.675 & 1.850
\end{array}\right], \\
T_{3}= & {\left[\begin{array}{llll}
1.800 & 1.800 & 1.800 & 1.875 \\
1.850 & 1.700 & 1.650 & 1.750 \\
1.825 & 1.850 & 1.700 & 1.825 \\
1.725 & 1.650 & 1.800 & 1.800
\end{array}\right] . }
\end{aligned}
$$

Step 5 Calculate the correlation weight $\varphi^{l}=\left[\varphi_{j k}^{l}\right]_{4 \times 4}$ based on the weight $\gamma_{l}$ of the decision maker $P_{l}$, and the results $\varphi^{\mathrm{l}}(l=1,2,3)$ are as follows:

$$
\begin{aligned}
\varphi^{1} & =\left[\begin{array}{llll}
0.3206 & 0.3181 & 0.3239 & 0.3244 \\
0.3278 & 0.3213 & 0.3154 & 0.3187 \\
0.3251 & 0.3238 & 0.3148 & 0.3251 \\
0.3168 & 0.3287 & 0.3206 & 0.3136
\end{array}\right], \\
\varphi^{2} & =\left[\begin{array}{llll}
0.4426 & 0.4553 & 0.4390 & 0.4444 \\
0.4367 & 0.4519 & 0.4601 & 0.4562 \\
0.4453 & 0.4475 & 0.4590 & 0.4453 \\
0.4576 & 0.4456 & 0.4426 & 0.4570
\end{array}\right], \\
\varphi^{3} & =\left[\begin{array}{llll}
0.2368 & 0.2266 & 0.2371 & 0.2312 \\
0.2355 & 0.2268 & 0.2245 & 0.2251 \\
0.2296 & 0.2287 & 0.2262 & 0.2296 \\
0.2256 & 0.2256 & 0.2368 & 0.2295
\end{array}\right] .
\end{aligned}
$$

Step 6 The BIs decision matrices obtained from Step 2 are merged into a decision matrix via using the weight matrix $\varphi^{l}$ in the IVIFWPMM $M_{D S T}$ operator $(\mathrm{s}=[1,1,1,1])$. The merged decision matrix is shown in Table 5 . 
Table 5. Merged BIs decision matrix.

\begin{tabular}{ccccc}
\hline & $r_{1}$ & $r_{2}$ & $r_{3}$ & $r_{4}$ \\
\hline \multirow{2}{*}{$t_{1}$} & $([0.2041,0.2374]$, & $([0.1561,0.2409]$, & $([0.1628,0.2374]$, & $([0.1852,0.2481]$, \\
& $[0.2295,0.2707])$ & $[0.2151,0.2894])$ & $[0.2149,0.2854])$ & $[0.2481,0.2923])$ \\
\multirow{2}{*}{$t_{2}$} & $([0.2042,0.2521]$, & $([0.1742,0.2365]$, & $([0.1531,0.2303]$, & $([0.2031,0.2592]$, \\
& $[0.2376,0.2854])$ & $[0.2075,0.2698])$ & $[0.1816,0.2636])$ & $[0.2365,0.2925])\}$ \\
\multirow{2}{*}{$t_{3}$} & $([0.1486,0.2482]$, & $([0.1816,0.2590]$, & $([0.1637,0.2304]$, & $([0.1815,0.2257]$, \\
& $[0.1852,0.2815])$ & $[0.2482,0.2924])$ & $[0.2304,0.2637])$ & $[0.2148,0.2739])$ \\
\multirow{2}{*}{$t_{4}$} & $([0.1697,0.2258]$, & $([0.1593,0.2260]$, & $([0.1707,0.2374]$, & $([0.1715,0.2562]$, \\
& $[0.2030,0.2697])$ & $[0.2002,0.2593])$ & $[0.2188,0.2814])$ & $[0.2562,0.2895])$ \\
\hline
\end{tabular}

Step 7 Calculate the support between $\widetilde{g_{j k}}$ and $\widetilde{g_{j l}}$, i.e., Sup $\left(\widetilde{g_{j k}}, \widetilde{g_{j l}}\right)=1-d\left(\widetilde{g_{j k}}, \widetilde{g_{j l}}\right)(j, k, l=1,2,3,4 ; l \neq k)$. Let $S_{k l}=\operatorname{Sup}\left(\widetilde{g_{j k}}, \widetilde{g_{j l}}\right)(j, k, l=1,2,3,4 ; l \neq k)$ for convenience, then the results are as follows:

$$
\begin{array}{ll}
S_{12}=S_{21}=(0.9788,0.9771,0.9706,0.9941) & S_{13}=S_{31}=(0.9823,0.9623,0.9760,0.9900) \\
S_{14}=S_{41}=(0.9825,0.9959,0.9768,0.9937) & S_{23}=S_{32}=(0.9964,0.9852,0.9767,0.9841) \\
S_{24}=S_{42}=(0.9819,0.9742,0.9787,0.9678) & S_{34}=S_{43}=(0.9817,0.9594,0.9880,0.9837)
\end{array}
$$

Step 8 Calculate $T_{j k}=T\left(\widetilde{g_{j k}}\right)$, then $T=\left[T_{j k}\right]_{4 \times 4}$ is as follows:

$$
T=\left[\begin{array}{llll}
2.9438 & 2.9571 & 2.9604 & 2.9461 \\
2.9354 & 2.9365 & 2.9609 & 2.9295 \\
2.9234 & 2.9260 & 2.9407 & 2.9434 \\
2.9577 & 2.9460 & 2.9578 & 2.9253
\end{array}\right] .
$$

Step 9 Calculate the weight $\delta_{j k}=\left[\delta_{j k}\right]$ corresponding to the BIs $\widetilde{g_{j k}}$, then $\delta=\left[\delta_{i j}\right]_{4 \times 4}$ is as follows:

$$
\delta=\left[\begin{array}{llll}
0.2994 & 0.4006 & 0.1002 & 0.1997 \\
0.3003 & 0.4005 & 0.0994 & 0.1999 \\
0.2995 & 0.3996 & 0.1003 & 0.2007 \\
0.3008 & 0.3999 & 0.1003 & 0.1989
\end{array}\right] .
$$

Step 10 Use the IVIFWPMM $M_{D S T}$ operator to calculate the comprehensive value $\widetilde{\mathrm{t}_{j}}(j=1,2,3,4)$ of each alternative (s $=$ $[1,1,1,1])$, and the results are as follows:

$$
\begin{aligned}
& \widetilde{t_{1}}=[(0.1559,0.1613),(0.1491,0.1485)], \widetilde{t_{2}}=[(0.2133,0.2160),(0.2131,0.2090)], \\
& \widetilde{t_{3}}=[(0.2006,0.1897),(0.1935,0.1934)], \widetilde{t_{4}}=[(0.2518,0.2455),(0.2460,0.2433)] .
\end{aligned}
$$

Step 11 Calculate $S_{j}=S F_{D S T}\left(\widetilde{\mathrm{t}_{j}}\right)(j=1,2,3,4)$, and we can obtain:

$$
S_{1}=-0.589, S_{2}=-0.594, S_{3}=-0.599, S_{4}=-0.603 \text {. }
$$

Step 12 According to the scores $S_{j}(j=1,2,3,4)$ we can get a ranking of all alternatives, that is: $t_{1}>t_{2}>t_{3}>t_{4}$. Thus, the best alternative is $t_{1}$.

\subsection{Effect of the parameters in $s$ on ranking results}

Section 5.1 shows that, parameters in $s$ are important variables in the decision-making process. To this end, to show the influence of parameter vector $s$ on decision-making results, we will use $s$ set by variant values to sort the alternatives in the example in Section 5.1. The ranking results obtained by setting $s$ with different values are shown in Table 6.

Table 6. Ranking orders with different $\mathrm{S}$ values in the IVIFWPMMDST operator.

\begin{tabular}{cll}
\hline$s$ & $S_{j}=S F_{D S T}\left(\widetilde{\mathrm{t}_{j}}\right), j=1,2,3,4$ & Ranking Orders \\
\hline$s=[1,1,1,1]$ & $S_{1}=-0.5892, S_{2}=-0.5938, S_{3}=-0.5991, S_{4}=-0.6029$ & $t_{1}>t_{2}>t_{3}>t_{4}$ \\
$s=[1,1,1,0]$ & $S_{1}=-0.5685, S_{2}=-0.5695, S_{3}=-0.5781, S_{4}=-0.5853$ & $t_{1}>t_{2}>t_{3}>t_{4}$ \\
$s=[1,1,1,0]$ & $S_{1}=-0.5512, S_{2}=-0.5505, S_{3}=-0.5588, S_{4}=-0.5707$ & $t_{2}>t_{1}>t_{3}>t_{4}$ \\
$S=[1,0,0,0]$ & $S_{1}=-0.5361, S_{2}=-0.5349, S_{3}=-0.5402, S_{4}=-0.5581$ & $t_{2}>t_{1}>t_{3}>t_{4}$ \\
\hline
\end{tabular}

Since the parameter vector $s$ represents the concern degree of the decision maker on different criteria (Xu, Shang, Wang \& Li, 2019), so the comprehensive evaluation values and rankings obtained by the IVIFWPMM DST $_{\text {operator in }}$ different parameter setting are also different, as shown in Table 6. Further, when less correlated attributes are considered by decision maker, the score of comprehensive evaluation value increases. Therefore, when making decisions, a more appropriate parameter s can be set according to the actual situation and actual demand. 


\subsection{Verification of the effectiveness}

In Section 5.1, we gave a brief demonstration of the proposed method through an example of selecting an optimal smart device scheme. In Section 5.2, we analyzed the influence of parameter vector $s$ on decision-making results. Next, we will demonstrate the effectiveness of the proposed method via a set of comparisons.

Example 5.2 (Sun, Gang \& Xia, 2016) A company needs to purchase office computers for employees working in a new office building. There are currently five computer brands and models $t_{i}(i=1,2,3,4,5)$ as possible alternatives. To select a more suitable alternative, the company needs to evaluate the five alternatives from the four attributes, i.e., computer price $\left(r_{1}\right)$, hardware performance $\left(r_{2}\right)$, after-sales service $\left(r_{3}\right)$, and service life $\left(r_{4}\right)$. A weight vector w $=$ $(0.2,0.1,0.3,0.4)^{\mathrm{T}}$ denotes the importance of each attribute. The company uses IVIFVs $g_{i j}=\left(\left[a_{i j}, b_{i j}\right],\left[c_{i j}, d_{i j}\right]\right)$ to evaluate the attribute $r_{j}$ of the alternative $t_{i}$. The decision matrix composed of IVIFVs $g_{i j}$ is shown in Table 7.

Table 7. The IVIFV decision matrix.

\begin{tabular}{ccccc}
\hline & $r_{1}$ & $r_{2}$ & $r_{3}$ & $r_{4}$ \\
\hline$t_{1}$ & $([0.4,0.5],[0.3,0.4])$ & $([0.4,0.6],[0.2,0.4])$ & $([0.1,0.3],[0.5,0.6])$ & $([0.3,0.4],[0.3,0.5])$ \\
$t_{2}$ & $([0.6,0.7],[0.2,0.3])$ & $([0.6,0.7],[0.2,0.3])$ & $([0.4,0.7],[0.1,0.2])$ & $([0.5,0.6],[0.1,0.3])$ \\
$t_{3}$ & $([0.3,0.6],[0.3,0.4])$ & $([0.5,0.6],[0.3,0.4])$ & $([0.5,0.6],[0.1,0.3])$ & $([0.4,0.5],[0.2,0.4])$ \\
$t_{4}$ & $([0.7,0.8],[0.1,0.2])$ & $([0.6,0.7],[0.1,0.3])$ & $([0.3,0.4],[0.1,0.2])$ & $([0.3,0.7],[0.1,0.2])$ \\
$t_{5}$ & $([0.3,0.4],[0.2,0.3])$ & $([0.3,0.5],[0.1,0.3])$ & $([0.2,0.5],[0.4,0.5])$ & $([0.3,0.4],[0.5,0.6])$ \\
\hline
\end{tabular}

Table 8. Ranking results of different methods in Example 5.2.

\begin{tabular}{|c|c|c|}
\hline Methods & Score Values & Ranking Orders \\
\hline IVIFWA (He et al., 2013) & $\begin{array}{l}S_{1}=-0.0661, S_{2}=0.3904, S_{3}=0.2185 \\
S_{4}=0.3962, S_{5}=-0.0396\end{array}$ & $t_{4}>t_{2}>t_{3}>t_{5}>t_{1}$ \\
\hline $\operatorname{IVIFPWA}(\mathrm{Xu}, 2007)$ & $\begin{array}{l}S_{1}=-0.5178, S_{2}=-0.2335, S_{3}=-0.3945 \\
S_{4}=-0.1933, S_{5}=-0.6314\end{array}$ & $t_{4}>t_{2}>t_{3}>t_{1}>t_{5}$ \\
\hline $\operatorname{IVIFWBM}(\mathrm{Xu} \&$ Chen, 2011) & $\begin{array}{l}S_{1}=-0.7085, S_{2}=-0.4866, S_{3}=-0.5854, \\
S_{4}=-0.4648, S_{5}=-0.6912\end{array}$ & $t_{4}>t_{2}>t_{3}>t_{5}>t_{1}$ \\
\hline GIVIFWHM (Yu \& Wu, 2012) & $\begin{array}{l}S_{1}=-0.6965, S_{2}=-0.4602, S_{3}=-0.5606 \\
S_{4}=-0.4382, S_{5}=-0.6846\end{array}$ & $t_{4}>t_{2}>t_{3}>t_{5}>t_{1}$ \\
\hline $\operatorname{IVIFWMSM}($ Sun \& Xia, 2016) & $\begin{array}{l}S_{1}=-0.7085, S_{2}=-0.4866, S_{3}=-0.5854, \\
S_{4}=-0.4648 S_{5}=-0.6912\end{array}$ & $t_{4}>t_{2}>t_{3}>t_{5}>t_{1}$ \\
\hline IVIFWPBM (Liu \& Li, 2017) & $\begin{array}{l}S_{1}=-0.1075, S_{2}=0.3037, S_{3}=0.1401 \\
S_{4}=0.3156, S_{5}=-0.0614\end{array}$ & $t_{4}>t_{2}>t_{3}>t_{5}>t_{1}$ \\
\hline IVIFWPHM (Liu, 2017) & $\begin{array}{l}S_{1}=-0.076, S_{2}=0.3407, S_{3}=0.1866 \\
S_{4}=0.3604 S_{5}=-0.0443\end{array}$ & $t_{4}>t_{2}>t_{3}>t_{1}>t_{5}$ \\
\hline IVIFWPMSM(Liu et al., 2018) & $\begin{array}{l}S_{1}=-0.1075, S_{2}=0.3037, S_{3}=0.1401, \\
S_{4}=0.3156, S_{5}=-0.0614\end{array}$ & $t_{4}>t_{2}>t_{3}>t_{5}>t_{1}$ \\
\hline $\begin{array}{l}\text { IVIFWPMM (Xu, Shang, Wang } \\
\text { \& Li, 2019) }\end{array}$ & $\begin{array}{l}S_{1}=0.6069, S_{2}=0.7685, S_{3}=0.6977 \\
S_{4}=0.7758, S_{5}=0.6164\end{array}$ & $t_{4}>t_{2}>t_{3}>t_{5}>t_{1}$ \\
\hline IVIFWMM (Xu et al., 2019) & $\begin{array}{l}S_{1}=-0.6349, S_{2}=-0.3882, S_{3}=-0.5071, \\
S_{4}=-0.3665, S_{5}=-0.6115\end{array}$ & $t_{4}>t_{2}>t_{3}>t_{5}>t_{1}$ \\
\hline$I V I F W P M M_{D S T}$ (ours) & $\begin{array}{l}S_{1}=0.0877, S_{2}=0.6042, S_{3}=0.4122, \\
S_{4}=0.6089, S_{5}=0.0256\end{array}$ & $t_{4}>t_{2}>t_{3}>t_{1}>t_{5}$ \\
\hline
\end{tabular}

Since there is no ranking by an authoritative expert for Example 5.2, we use the ranking results of multiple diachronically tested MCDM methods (Xu, 2007; He et al., 2013; Sun, Gang \& Xia, 2016; Liu \& Li, 2017; Yu \& Wu, 2012; Xu \& Chen, 2011; Liu, 2017; Liu et al., 2018) to verify the effectiveness of the presented MCDM method . Specifically, we use the ranking results of the following operators, i.e., the interval-valued intuitionistic fuzzy weighted average (IVIFWA) operator (He et al., 2013), the interval-valued intuitionistic fuzzy power weighted average (IVIFPWA) operator (Xu, 2007)[8], the interval-valued intuitionistic fuzzy weighted average weighted bonferroni mean (IVIFWBM) operator (Xu \& Chen, 2011), the generalized interval-valued intuitionistic fuzzy weighted Heronian mean (GIVIFWHM) operator (Yu \& Wu, 2012), and the interval-valued intuitionistic fuzzy weighted Maclaurin symmetric mean (IVIFWMSM) operator (Sun \& Xia, 2016), the interval-valued intuitionistic fuzzy weighted power Bonferroni mean 
(IVIFWPBM) operator (Liu \& Li, 2017), the interval-valued intuitionistic fuzzy weighted power Heronian mean (IVIFWPHM) operator (Liu, 2017), and the interval-valued intuitionistic fuzzy weighted Maclaurin symmetric mean (IVIFWPMSM) operator (Liu et al., 2018). As shown in Table 8, the ranking result of our operator is the same as that of most existing operators. This verifies the feasibility and effectiveness of the proposed method .

\subsection{Further comparative analysis}

From the results of Section 5.3, we can demonstrate the effectiveness of the proposed method. However, since almost all methods have the same sorting results, the limitations of the existing methods and the advantages of the proposed method cannot be seen from the comparison in Section 5.3. To this end, we will illustrate the advantages of our method via further comparisons The methods based on IVIFPWA (Xu, 2007) and IVIFWA (He et al., 2013) are selected as comparison objects.

\subsubsection{Comparison of robustness}

In MCDM methods without human intervention, unreasonable decision values may be resulted by data transmission failure, decision makers' preferences or other accidents. In this case, it may be difficult to obtain a reasonable sorting result via these methods. The robustness of the proposed method is evaluated via Example 5.3.

Example 5.3 To simulate the situation with extreme evaluation values, the value of $g_{42}=([0.6,0.7],[0.1,0.3])$ in Example 5.2 is changed to $g_{42}=([0.01,0.7],[0.1,0.3])$. Then, the new decision matrix and the ranking results are shown in Table 9 and Table 10, respectively.

Table 9. The IVIFV decision matrix.

\begin{tabular}{ccccc}
\hline \multicolumn{7}{c}{$r_{1}$} & $r_{2}$ & $r_{3}$ & $r_{4}$ \\
\hline$t_{1}$ & $([0.4,0.5],[0.3,0.4])$ & $([0.4,0.6],[0.2,0.4])$ & $([0.1,0.3],[0.5,0.6])$ & $([0.3,0.4],[0.3,0.5])$ \\
$t_{2}$ & $([0.6,0.7],[0.2,0.3])$ & $([0.6,0.7],[0.2,0.3])$ & $([0.4,0.7],[0.1,0.2])$ & $([0.5,0.6],[0.1,0.3])$ \\
$t_{3}$ & $([0.3,0.6],[0.3,0.4])$ & $([0.5,0.6],[0.3,0.4])$ & $([0.5,0.6],[0.1,0.3])$ & $([0.4,0.5],[0.2,0.4])$ \\
$t_{4}$ & $([0.7,0.8],[0.1,0.2])$ & $([0.01,0.7],[0.1,0.3])$ & $([0.3,0.4],[0.1,0.2])$ & $([0.3,0.7],[0.1,0.2])$ \\
$t_{5}$ & $([0.3,0.4],[0.2,0.3])$ & $([0.3,0.5],[0.1,0.3])$ & $([0.2,0.5],[0.4,0.5])$ & $([0.3,0.4],[0.5,0.6])$ \\
\hline
\end{tabular}

Table 10. Ranking results of different methods in Example 5.3.

\begin{tabular}{clc}
\hline Methods & \multicolumn{1}{c}{ Score Values } & Ranking Orders \\
\hline IVIFPWA (Xu, 2007) & $S_{1}=-0.0607, S_{2}=0.3172, S_{3}=0.2179$, & $t_{4}>t_{2}>t_{3}>t_{5}>t_{1}$ \\
& $S_{4}=0.3669, S_{5}=-0.0384$ & \\
\hline IVIFWA (He et al., 2013) & $S_{1}=-0.0661, S_{2}=0.3904, S_{3}=0.2185$, & $t_{2}>t_{4}>t_{3}>t_{5}>t_{1}$ \\
& $S_{4}=0.3103, S_{5}=-0.0396$ & \\
\hline IVIFWMM (Xu et al., 2019) & $S_{1}=-0.0589, S_{2}=0.3819, S_{3}=0.1919$, & $t_{2}>t_{4}>t_{3}>t_{5}>t_{1}$ \\
& $S_{4}=0.2307, S_{5}=-0.0202$ & $t_{4}>t_{2}>t_{3}>t_{5}>t_{1}$ \\
\hline IVIFVWPMM
\end{tabular}

As shown in Table 10, the extreme value $g_{42}=([0.01,0.7],[0.1,0.3])$ has an obvious impact on the sorting results of the operators IVIFWA (He et al., 2013) and IVIFWMM: the sorting results of these two operators both have changed from the original result of $t_{4}>t_{2}>t_{3}>t_{5}>t_{1}$ to $t_{2}>t_{4}>t_{3}>t_{5}>t_{1}$. Accordingly, IVIFWA (He et al., 2013) and IVIFWMM operators are not robust to extreme value such that they get unreasonable sorting results in this case. Therefore, two operators may not get the reasonable ranking results in an unstable system (e.g., with abnormal decision values), when there is no intervention of experts. In contrast, the IVIFVWPMM can get reasonable sorting results. This is because the $P A$ operator calculate the distance among criteria, and then the weight of each criterion is obtained by this distance. Therefore, unreasonable opinions are assigned to a smaller weight, thus reducing the negative effect of these unreasonable opinions to the final sorting results. The proposed operator is well integrated with the $P A$ operator under the DST framework and therefore inherits the advantage of the $P A$ operator to reduce the negative effect of extreme values.

5.4.2. Comparison of the running time and space

In real-time MCDM problems, running time and space are important factors for evaluating an operator. To compare 
the running time and space of different operators, we test Example 5.210 times in the same environment and then averages the running time and space of each operator to get the results in Table 11.

Table 11. Comparisons of MCDM methods on time and space.

\begin{tabular}{ccc}
\hline Methods & Running Time & Running space \\
\hline IVIFPWA (Xu, 2007) & $0.02 \mathrm{~s}$ & $530 \mathrm{~kb}$ \\
\hline$I V I F W A(\mathrm{He}$ et al., 2013) & $0.02 \mathrm{~s}$ & $560 \mathrm{~kb}$ \\
\hline IVIFWMM (Xu et al., 2019) & $0.05 \mathrm{~s}$ & $856 \mathrm{~kb}$ \\
\hline$I V I F W P M M_{D S T}$ (our) & $0.04 \mathrm{~s}$ & $532 \mathrm{~kb}$ \\
\hline
\end{tabular}

As shown in Table 11, the proposed IVIFVWPMM $M_{D S T}$ operator has a longer running time than the IVIFWA and IVIFPWA operators, but the running space has no obvious difference. This is because calculating the Muirhead mean in IVIFVWPMM $M_{D S T}$ increases the time and space complexity.However, compared with the base IVIFWMM operator that also calculates the Muirhead mean, the proposed operator requires less running time and space. This is due to the less time and space complexity of IVIFV under the DST framework. Therefore, IVIFVWPMM $M_{D S T}$ operator outperforms the IVIFWMM operator.

\subsubsection{Comparison of form of weights}

In actual MCDM problems, there exist a case that expert weights $\gamma$ or attribute weights $w$ are not exact positive numbers. In this case, defining the weights as real numbers may lead to unreasonable ranking results. To this end, the weights can be defined as IVIFVs so that the rationality of the ranking results can be improved (Liu, 2017; Zhong et al., 2021).

Example 5.4 To simulate this situation, we replace the positive number weights $w=(0.2,0.1,0.3,0.4)^{\mathrm{T}}$ in Example 5.2 with the interval fuzzy values $w=[0.05,0.15,0.1,0.4 ; 0,0.1,0.1,0.2 ; 0.1,0.3,0.3,0.5 ; 0.4,0.6,0.6,0.8]$

$([0.1,0.3],[0.05,0.15],[0.2,0.4],[0.3,0.5])$. Table 12 lists the sorting results of several related methods. As shown in Table 12, the proposed operator expands the scope of application of the original operator to the case where the weights are IVIFVs.

Table 12. Ranking results of different methods in Example 5.4.

\begin{tabular}{ccc}
\hline Methods & Score Values & Ranking Orders \\
\hline IVIFPWA $(\mathrm{Xu}, 2007)$ & Cannot be counted & Cannot be ranked \\
\hline IVIFWA $(\mathrm{He}$ et al.2013) & Cannot be counted & Cannot be ranked \\
\hline IVIFWMM $(\mathrm{Xu}$ et al., 2019) & Cannot be counted & Cannot be ranked \\
\hline IVIFVWPMM $M_{D S T}$ (ours) & $S_{1}=-0.5884, S_{2}=-0.4275, S_{3}=-0.5001$, & $t_{4}>t_{2}>t_{3}>t_{1}>t_{5}$ \\
& $S_{4}=-0.4083, S_{5}=-0.6402$ & \\
\hline
\end{tabular}

\subsubsection{Summary of the comparisons}

All in all, through Examples 5.2 - 5.4, we can further summarize the following limitations of the IVIFPWA operator (Xu, 2007), IVIFWA operator (He et al., 2013) and IVIFWMM operator: (1) The IVIFPWA operator does not perform the operation for eliminating parameter correlation, and secondly, it cannot handle the case that the decision weights are IVIFVs. (2) Similarly, the IVIFWA operator (He et al., 2013) also has the above two limitations like IVIFPWA operator $(\mathrm{Xu}, 2007)$. At the same time, this operator cannot reduce the weight of abnormal opinions via the distance among criteria, which may result in unreasonable sorting results. (3) The IVIFWMM operator has the limitation that the weights cannot be adjusted according to the existing information, so it cannot deal with the data distortion in the unstable system or extreme situation. Moreover, due to the high running time and space, the operator occupies more calculation time and space and cannot deal well with real-time MCDM problems. In addition, it also cannot handle the case that the decision weights are IVIFVs. To make the summary more intuitive, Table 13 lists the comparison between the proposed methods with several existing methods.

Table 13. Comparison results of the characteristics.

\begin{tabular}{cccc}
\hline Methods & $\begin{array}{c}\text { Whether eliminate } \\
\text { the effects of }\end{array}$ & $\begin{array}{l}\text { Whether criteria } \\
\text { weights can be }\end{array}$ & Running Time Running space \\
\hline
\end{tabular}




\begin{tabular}{|c|c|c|c|c|}
\hline & extreme values & denoted by IFNs & & \\
\hline IVIFPWA (Xu, 2007) & Yes & No & $0.02 \mathrm{~s}$ & $530 \mathrm{~kb}$ \\
\hline IVIFWA (He et al., 2013) & No & No & $0.02 \mathrm{~s}$ & $560 \mathrm{~kb}$ \\
\hline $\operatorname{IVIFWMM}(\mathrm{Xu}$ et al., 2019) & No & No & $0.05 \mathrm{~s}$ & $856 \mathrm{~kb}$ \\
\hline$I V I F V W P M M_{D S T}$ (ours) & Yes & Yes & $0.04 \mathrm{~s}$ & $532 \mathrm{~kb}$ \\
\hline
\end{tabular}

\section{Conclusions}

In this paper, we firstly propose two operators of IVIFVs under the DST framework which are free of the limitations of existing aggregation operators of IVIFVs. Subsequently, the properties of these operators are discussed and proved. Then, we present a method based on the proposed operators, to solve the MCDM problems with IVIFVs. Finally, a set of practical examples are introduced to illustrate the application of the presented method and demonstrate the effectiveness, robustness, and advantages of the presented method. The demonstration results show that: The criterion weights or expert weights can be expressed by IVIFVs in the presented method; The presented method can eliminate parameter correlation; The sorting results of the presented method are robust to the abnormal decision values; The presented method just needs less running space and comparable time. In the future, we will combine the proposed operators with deep learning or reinforcement learning, and employ them to other fields, such as image quality assessment, image aesthetic assessment, and underwater robot path planning.

\section{Compliance with ethical standards}

Conflict of interest The authors declare that they have no conflict of interest.

\section{Acknowledgements}

The authors would like to thank the pioneering researchers in fuzzy decision and other related fields. The authors $\mathrm{w}$ ould also like to express their sincere appreciation to the associate editor and the anonymous reviewers for their commen ts and suggestions. This work has been supported by the National Natural Science Foundation of China under Grant 621 66011 and 62033001.

\section{References}

Atanassov, K. T. (1999). Interval valued intuitionistic fuzzy sets. In Intuitionistic Fuzzy Sets (pp. 139-177). Physica, Heidelberg.

Atanassov, K. T. (1986). Intuitionistic fuzzy sets. Fuzzy Sets and Systems, 20(1), 87-96.

Blanco-Mesa, F., Merigó, J. M., \& Gil-Lafuente, A. M. (2017). Fuzzy decision making: A bibliometric-based review. Journal of Intelligent \& Fuzzy Systems, 32(3), 2033-2050.

Chen, S. M., \& Kuo, L. W. (2018). Multiattribute decision making based on non-linear programming methodology with hyperbolic function and interval-valued intuitionistic fuzzy values. Information Sciences, 453, 379-388.

Chen, S. M., \& Tsai, K. Y. (2021). Multiattribute decision making based on new score function of interval-valued intuitionistic fuzzy values and normalized score matrices.Information Sciences, 575, 714-731.

Chen, T. Y. (2015). The inclusion-based TOPSIS method with interval-valued intuitionistic fuzzy sets for multiple criteria group decision making. Applied Soft Computing, 26, 57-73.

Dempster, A. P. (1967). Upper and lower probabilities induced by a multivalued mapping. The Annals of Mathematical Statistics, 38(2), 325-339. 
Dempster, A. P. (2008). Upper and lower probabilities induced by a multivalued mapping. In Classic works of the Dempster-Shafer theory of belief functions (pp. 57-72). Springer, Berlin, Heidelberg.

Dymova, L., \& Sevastjanov, P. (2010). An interpretation of intuitionistic fuzzy sets in terms of evidence theory: decision making aspect. Knowledge-Based Systems, 23(8), 772-782.

Dymova, L., \& Sevastjanov, P. (2012). The operations on intuitionistic fuzzy values in the framework of DempsterShafer theory. Knowledge-Based Systems, 35, 132-143.

Dymova, L., \& Sevastjanov, P. (2016). The operations on interval-valued intuitionistic fuzzy values in the framework of Dempster-Shafer theory. Information Sciences, 360, 256-272.

Einstein, A., Podolsky, B., \& Rosen, N. (1935). Can quantum-mechanical description of physical reality be considered complete?. Physical review, 47(10), 777.

Gao, H., Zhang, H., \& Liu, P. (2019). Multi-attribute decision making based on intuitionistic fuzzy power Maclaurin symmetric mean operators in the framework of Dempster-Shafer theory. Symmetry, 11(6), 807.

Garg, H., Mahmood, T., Ahmmad, J., Khan, Q., \& Ali, Z. (2021). Cubic q-Rung Orthopair Fuzzy Linguistic Set and Their Application to Multiattribute Decision-making with Muirhead Mean Operator. Journal of Artificial Intelligence and Technology, 1(1), 37-50.

Garg, H., \& Arora, R. (2018). A nonlinear-programming methodology for multi-attribute decision-making problem with interval-valued intuitionistic fuzzy soft sets information.Applied Intelligence, 48(8), 2031-2046.

Garg, H., \& Kaur, G. (2019). TOPSIS based on nonlinear-programming methodology for solving decision-making problems under cubic intuitionistic fuzzy set environment.Computational and Applied Mathematics, 38(3), 1-19.

Garg, H., \& Kumar, K. (2020). A novel exponential distance and its based TOPSIS method for interval-valued intuitionistic fuzzy sets using connection number of SPA theory. Artificial Intelligence Review, 53(1), 595-624.

Garg, H., \& Kumar, K. (2020). A novel possibility measure to interval-valued intuitionistic fuzzy set using connection number of set pair analysis and its applications. Neural Computing and Applications, 32(8), 3337-3348.

Greco, S., Figueira, J., \& Ehrgott, M. (2016). Multiple criteria decision analysis: State of the art surveys. New York: Springer-Verlag New York.

He, Y., Chen, H., Zhou, L., Liu, J., \& Tao, Z. (2013). Generalized Interval-Valued Atanassov's Intuitionistic Fuzzy Power Operators and Their Application to Group Decision Making. International Journal of Fuzzy Systems, 15(4).

Hung, W. L., \& Wu, J. W. (2002). Correlation of intuitionistic fuzzy sets by centroid method. Information sciences, 144(1-4), 219-225.

Jianfang, F. U., Tao, M. I. A. O., \& Keqin, W. U. (2021). A Modified Comprehensive Evaluation System of Groundwater Pollution Based on Fuzzy Set Theory. Meteorological \& Environmental Research, 12(1).

Khan, Q., Hassan, N., \& Mahmood, T. (2018). Neutrosophic cubic power Muirhead mean operators with uncertain data for multi-attribute decision-making. Symmetry, 10(10), 444.

Kumar, K., \& Chen, S. M. (2021). Multiattribute decision making based on interval-valued intuitionistic fuzzy values, score function of connection numbers, and the set pair analysis theory. Information Sciences, 551, 100-112.

Liu, B., Guo, S., Yan, K., Li, L., \& Wang, X. (2017). Double weight determination method for experts of complex multiattribute large-group decision-making in interval-valued intuitionistic fuzzy environment. Journal of Systems Engineering and Electronics, 28(1), 88-96.

Li, C., \& Jiang, H. (2011, August). Extension of VIKOR method with interval-valued intuitionistic fuzzy sets. In 2011 International Conference on Management and Service Science (pp. 1-4). IEEE.

Liu, P., Chen, S. M., \& Wang, P. (2018). Multiple-attribute group decision-making based on q-rung orthopair fuzzy power maclaurin symmetric mean operators. IEEE Transactions on Systems, Man, and Cybernetics: Systems, 50(10), 3741-3756. 
Liu, P., \& Gao, H. (2019). Some intuitionistic fuzzy power Bonferroni mean operators in the framework of DempsterShafer theory and their application to multicriteria decision making. Applied Soft Computing, 85, Article 105790.

Liu, P., \& Li, H. (2017). Interval-valued intuitionistic fuzzy power Bonferroni aggregation operators and their application to group decision making. Cognitive Computation, 9(4), 494-512.

Liu, P., \& Liu, W. (2019). Multiple-attribute group decision-making method of linguistic q-rung orthopair fuzzy power Muirhead mean operators based on entropy weight.International Journal of Intelligent Systems, 34(8), 1755-1794.

Liu, P. (2017). Multiple attribute group decision making method based on interval-valued intuitionistic fuzzy power Heronian aggregation operators. Computers \& Industrial Engineering, 108, 199-212.

Liu, Z., Teng, F., Liu, P., \& Ge, Q. (2018). Interval-valued intuitionistic fuzzy power Maclaurin symmetric mean aggregation operators and their application to multiple attribute group decision-making. International Journal for Uncertainty Quantification, 8(3).

Li, L., Mao, C., Lei, B., Gao, Y., Liu, Y., \& Huang, G. Q. (2020). Decision-making of product-service system solution selection based on integrated weight and technique for order preference by similarity to an ideal solution. IET Collaborative Intelligent Manufacturing, 2(3), 102-108.

Meng, F., Chen, S. M., \& Tang, J. (2021). Multicriteria decision making based on bi-direction Choquet integrals.Information Sciences, 555, 339-356.

Muirhead, R. F. (1902). Some methods applicable to identities and inequalities of symmetric algebraic functions of $n$ letters.Proceedings of the Edinburgh Mathematical Society, 21, 144-162.

Oztaysi, B., Onar, S. C., Goztepe, K., \& Kahraman, C. (2017). Evaluation of research proposals for grant funding using interval-valued intuitionistic fuzzy sets. Soft Computing,21(5), 1203-1218.

Pedrycz, W., \& Chen, S. M. (Eds.). (2015). Granular computing and decision-making: interactive and iterative approaches (Vol. 10). Springer.

Pramanik, R., Baidya, D. K., \& Dhang, N. (2021). Reliability assessment of three-dimensional bearing capacity of shallow foundation using fuzzy set theory. Frontiers of Structural and Civil Engineering, 0 .

Qin, Y., Qi, Q., Shi, P., Scott, P. J., \& Jiang, X. (2020). Novel operational laws and power Muirhead mean operators of picture fuzzy values in the framework of Dempster-Shafer theory for multiple criteria decision making. Computers \& Industrial Engineering, 149, 106853.

Ren, H., \& Wang, G. (2015). An interval-valued intuitionistic fuzzy MADM method based on a new similarity measure.Information, 6(4), 880-894.

Shafer, G. (1976). A mathematical theory of evidence. Princeton university press.

Shen, Q., Huang, X., Liu, Y., Jiang, Y., \& Zhao, K. (2020). Multiattribute decision making based on the binary connection number in set pair analysis under an interval-valued intuitionistic fuzzy set environment. Soft Computing, 24(10), 7801-7809.

Sun, G., \& Xia, W. L. (2016). Evaluation method for innovation capability and efficiency of high technology enterprises with interval-valued intuitionistic fuzzy information.Journal of intelligent \& fuzzy systems, 31(3), 1419-1425.

Torra, V. (2010). Hesitant fuzzy sets. International Journal of Intelligent Systems, 25(6), 529-539.

Veeramachaneni, S., \& Kandikonda, H. (2016). An ELECTRE approach for multicriteria interval-valued intuitionistic trapezoidal fuzzy group decision making problems. Advances in Fuzzy systems, 2016.

Wang, Y. M., \& Elhag, T. M. (2006). On the normalization of interval and fuzzy weights. Fuzzy sets and systems, 157(18), 2456-2471.

$\mathrm{Xu}$, W., Shang, X., Wang, J., \& Li, W. (2019). A novel approach to multi-attribute group decision-making based on interval-valued intuitionistic fuzzy power Muirhead mean.Symmetry, 11(3), 441. 
$\mathrm{Xu}, \mathrm{Z}$. (2007). Intuitionistic fuzzy aggregation operators. IEEE Transactions on fuzzy systems, 15(6), 1179-1187.

$\mathrm{Xu}, \mathrm{Z}$. (2007). Methods for aggregating interval-valued intuitionistic fuzzy information and their application to decision making [J]. Control and decision, 2, 019.

$\mathrm{Xu}$, Z. (2009). Intuitionistic fuzzy hierarchical clustering algorithms. Journal of Systems Engineering and Electronics,20(1), 90-97.

$\mathrm{Xu}$, Z. (2010). A deviation-based approach to intuitionistic fuzzy multiple attribute group decision making. Group Decision and Negotiation, 19(1), 57-76.

$\mathrm{Xu}, \mathrm{Z}$., \& Chen, Q. (2011). A multi-criteria decision making procedure based on interval-valued intuitionistic fuzzy bonferroni means. Journal of Systems Science and Systems Engineering, 20(2), 217-228.

Xu, Z., \& Yager, R. R. (2009). Power-geometric operators and their use in group decision making. IEEE Transactions on Fuzzy Systems, 18(1), 94-105.

Yager, R. R. (2001). The power average operator. IEEE Transactions on Systems, Man, and Cybernetics-Part A: Systems and Humans, 31(6), 724-731.

Yu, D., \& Wu, Y. (2012). Interval-valued intuitionistic fuzzy Heronian mean operators and their application in multicriteria decision making. African Journal of Business Management,6(11), 4158-4168.

Zadeh, L. A., Klir, G. J., \& Yuan, B. (1996). Fuzzy sets, fuzzy logic, and fuzzy systems: selected papers (Vol. 6). World Scientific.

Zeng, S., Chen, S. M., \& Kuo, L. W. (2019). Multiattribute decision making based on novel score function of intuitionistic fuzzy values and modified VIKOR method. Information Sciences, 488, 76-92.

Zhang, L., Zhan, J., \& Yao, Y. (2020). Intuitionistic fuzzy TOPSIS method based on CVPIFRS models: An application to biomedical problems. Information Sciences, 517, 315-339.

Zhong, Y., Cao, L., Zhang, H., Qin, Y., Huang, M., \& Luo, X. (2021). Hesitant fuzzy power Maclaurin symmetric mean operators in the framework of Dempster-Shafer theory for multiple criteria decision making. Journal of Ambient Intelligence and Humanized Computing, 1-21.

Zindani, D., Maity, S. R., \& Bhowmik, S. (2020). Interval-valued intuitionistic fuzzy TODIM method based on Schweizer-Sklar power aggregation operators and their applications to group decision making. Soft Computing, 1-43. 